\title{
Composition limits and separating examples for some Boolean function complexity measures
}

\author{
Justin Gilmer* \\ Department of Mathematics \\ Rutgers University \\ Piscataway, NJ, USA. \\ jmgilmer@math.rutgers . edu \\ Michael Saks ${ }^{\dagger}$ \\ Department of Mathematics \\ Rutgers University \\ Piscataway, NJ, USA. \\ saks@math . rutgers . edu \\ Srikanth Srinivasan ${ }^{\ddagger}$ \\ Department of Mathematics \\ IIT Bombay \\ Mumbai, India. \\ srikanth@math.iitb.ac.in
}

October 31, 2018

\begin{abstract}
Block sensitivity $(b s(f))$, certificate complexity $(C(f))$ and fractional certificate complexity $\left(C^{*}(f)\right)$ are three fundamental combinatorial measures of complexity of a boolean function $f$. It has long been known that $b s(f) \leq C^{*}(f) \leq C(f)=O\left(b s(f)^{2}\right)$. We provide an infinite family of examples for which $C(f)$ grows quadratically in $C^{*}(f)$ (and also $b s(f)$ ) giving optimal separations between these measures. Previously the biggest separation known was $C(f)=$ $C^{*}(f)^{\log _{4.5} 5}$. We also give a family of examples for which $C^{*}(f)=\Omega\left(b s(f)^{3 / 2}\right)$.

These examples are obtained by composing boolean functions in various ways. Here the composition $f \circ g$ of $f$ with $g$ is obtained by substituting for each variable of $f$ a copy of $g$ on disjoint sets of variables. To construct and analyse these examples we systematically investigate the behaviour under function composition of these measures and also the sensitivity measure $s(f)$. The measures $s(f), C(f)$ and $C^{*}(f)$ behave nicely under composition: they are submultiplicative (where measure $m$ is submultiplicative if $m(f \circ g) \leq m(f) m(g)$ ) with equality holding under some fairly general conditions. The measure $b s(f)$ is qualitatively different: it is not submultiplicative. This qualitative difference was not noticed in the previous literature and we correct some errors that appeared in previous papers. We define the composition limit of a measure $m$ at function $f, m^{\lim }(f)$ to be the limit as $k$ grows of $m\left(f^{(k)}\right)^{1 / k}$, where $f^{(k)}$ is the iterated composition of $f$ with itself $k$-times. For any function $f$ we show that $b s^{\lim }(f)=$ $\left(C^{*}\right)^{\lim }(f)$ and characterize $s^{\lim }(f),\left(C^{*}\right)^{\lim }(f)$, and $C^{\lim }(f)$ in terms of the largest eigenvalue of a certain set of $2 \times 2$ matrices associated with $f$.
\end{abstract}

* Supported by NSF grant CCF 083727

${ }^{\dagger}$ Supported by NSF grants CCF-083727 and CCF-1218711

${ }^{\ddagger}$ Work partially done as a Postdoctoral researcher at DIMACS, Rutgers University. 


\section{Introduction}

\subsection{Measures, critical exponents and iterated limits}

There is a large class of complexity measures for boolean functions that seek to quantify, for each function $f$, the amount of knowledge about individual variables needed to evaluate $f$. These include decision tree complexity and its randomized and quantum variants, (Fourier) degree, certificate complexity, sensitivity, and block sensitivity. The value of such a measure is at most the number of variables. There is a long line of research aimed at bounding one such measure in terms of another. For measures $a$ and $b$ let us write $a \leq_{r} b$ if there are constants $C_{1}, C_{2}$ such that for every total boolean function $f, a(f) \leq C_{1} b(f)^{r}+C_{2}$. For example, the decision tree complexity of $f, D(f)$, is at least its degree $\operatorname{deg}(f)$ and thus $\operatorname{deg} \leq_{1} D$. It is also known Mid04 that $D \leq_{3} \operatorname{deg}$. We say that $a$ is polynomially bounded by $b$ if $a \leq_{r} b$ for some $r>0$ and that $a$ and $b$ are polynomially equivalent if each is polynomially bounded by the other. The measures mentioned above, with the notable exception of sensitivity, are known to be polynomially equivalent.

For a function $f$, the decision tree complexity, degree, certificate complexity, block sensitivity and sensitivity of $f$ are denoted, respectively, $D(f), \operatorname{deg}(f), C(f), b s(f)$ and $s(f)$. We also define the fractional certificate complexity $C^{*}(f)$ (which is within constant factors of the randomized certificate complexity defined in [Aar08]; see Appendix A]). These measures are defined in Section 2. definitions of others may be found in the survey [BdW02].

If measure $a$ is polynomially bounded by $b$ we define the critical exponent for $b$ relative to $a$, $\operatorname{crit}(a, b)$, to be the infimum $r$ such that $a \leq_{r} b$, which (essentially) gives the tightest possible upper bound of $a$ as a power of $b$. In [HKP11, there is a table giving the best known upper and lower bounds for the critical exponents for all pairs from degree, deterministic query complexity, certificate complexity and block sensitivity. For example it is known that $\operatorname{crit}(D, C)=2$, while for $\operatorname{crit}(D$, deg $)$ the best bounds known are $\log _{3}(6) \leq \operatorname{crit}(D$, deg $) \leq 3$. Typically, lower bounds on $\operatorname{crit}(a, b)$ (implicitly) use the following fact:

Proposition 1. Let $\left(f_{k}: k \geq 1\right)$ be a sequence of boolean functions for which $b\left(f_{k}\right)$ tends to infinity. If $\log a\left(f_{k}\right) / \log b\left(f_{k}\right)$ tends to a limit $s$ then $\operatorname{crit}(a, b) \geq s$. More generally,

$$
\operatorname{crit}(a, b) \geq \liminf \frac{\log a\left(f_{k}\right)}{\log b\left(f_{k}\right)}
$$

The proof of this proposition is routine. Useful lower bounds on $\operatorname{crit}(a, b)$ are obtained by carefully selecting the sequence $\left(f_{k}\right)$. One approach to choosing the sequence is to select some $f_{1}$ and define $f_{k}$ to be the $k$ th iterated composition of $f_{1}$, which is defined as follows. If $f$ and $g$ are boolean functions, respectively, on $n$ and $m$ variables then $f \circ g$ is defined on $n m$ variables split into $n$ blocks of $m$ variables and is obtained by evaluating $g$ on each block, and then evaluating $f$ on the sequence of $n$ outputs. The $k$ th iterated composition of $f$ is defined inductively by $f^{(1)}=f$ and $f^{(k)}=f \circ f^{(k-1)}$ for $k \geq 2$. We say that a complexity measure $a$ is multiplicative with respect to function $f$ if $a\left(f^{(k)}\right)=a(f)^{k}$ for all $k \geq 1$. We say that $a$ is multiplicative if for any two functions $f$ and $g$ we have $a(f \circ g)=a(f) a(g)$; this condition implies immediately that $a$ is multiplicative with respect to every function $f$. As a direct consequence of Proposition 1 we have:

Proposition 2. If $a$ and $b$ are complexity measures that are each multiplicative with respect to the function $f$ then $\operatorname{crit}(a, b) \geq \log a(f) / \log b(f)$. 
For example, the lower bound $\operatorname{crit}(D, \operatorname{deg}) \geq \log _{3} 6$ is obtained by applying Proposition 2 to a specific six variable boolean function $f$ having $\operatorname{deg}(f)=3$ and $D(f)=6$ using the easy fact that the measures $d e g$ and $D$ are multiplicative.

For non-multiplicative measures $a, b$ one may be able to identify specific functions $f$ such that $a$ and $b$ are each multiplicative on $f$ which is enough to use Proposition 2. While $s, C, C^{*}$ are not multiplicative, each is multiplicative on functions $f$ that satisfy $m_{0}(f)=m_{1}(f)$ (see Section 2.4 for definitions). However, this fails for block sensitivity, and this failure is responsible for some errors in the literature. In Aar08 it was proposed that a six variable function $f$ given by Paterson (see [BSW86]) could be used to obtain a lower bound on the critical exponent of block sensitivity relative to certificate complexity. The function $f$ has block sensitivity 4 and certificate complexity 5 and in fact satisfies $b s_{x}(f)=4$ and $C_{x}(f)=5$ for all inputs $x$. This was used to deduce that both block sensitivity and certificate complexity are multiplicative on $f$, and therefore $\operatorname{crit}(C, b s) \leq \log _{4} 5$. It turns out, however, that block sensitivity is not multiplicative with respect to $f$. In this case, $b s\left(f^{(m)}\right)^{1 / m}$ tends to 4.5 rather than 4 and so the resulting lower bound on $\operatorname{crit}\left(C^{*}, b s\right)$ is $\log _{4.5}(5)$ rather than $\log _{4} 5$.

Proposition 2 can be extended to the case that $a$ and $b$ are not necessarily multiplicative on $f$. Given any measure $a$ we can define a new measure $a^{\text {lim }}$, called the composition limit of $a$, where $a^{\lim }(f)=\liminf a\left(f^{(k)}\right)^{1 / k}$. If $a$ is multiplicative on $f$ then $a^{\lim }(f)=a(f)$. Applying Proposition 11 yields the following extension of Proposition 2,

Proposition 3. Suppose $a$ and $b$ are complexity measures and $f$ is a boolean function for which $b^{\lim }(f)>1$. Then $\operatorname{crit}(a, b) \geq \log a^{\lim }(f) / \log b^{\lim }(f)$.

To apply this proposition, we need to analyse $a^{\lim }(f)$ and $b^{\lim }(f)$.

\subsection{The contributions of this paper}

In this paper we analyse the behaviour of certificate complexity, fractional certificate complexity, sensitivity and block sensitivity under composition. This enables us to give characterizations of the composition limits $s^{\lim }, C^{\lim },\left(C^{*}\right)^{\lim }$ and $b s^{\lim }$. We also obtain new lower bounds on $\operatorname{crit}(C, b s)$, $\operatorname{crit}\left(C, C^{*}\right)$ and $\operatorname{crit}\left(C^{*}, b s\right)$; in the first two cases the new lower bounds are tight.

The paper is organized as follows.

- In Section 2 we give various definitions and technical preliminaries. We introduce a new notion of an assemblage, which provides a common abstraction for the objects underlying the measures $s(f), C(f), b s(f)$, and $C^{*}(f)$.

- In Section 3, we characterize the composition limit of $s(f), C(f)$ and $C^{*}(f)$. For $m \in$ $\left\{s(f), C(f), C^{*}(f)\right\}$, we always have $\min \left\{m_{0}(f), m_{1}(f)\right\} \leq m^{\lim }(f) \leq m(f)$. We express the composition limit as the minimum over a certain family of 2 by 2 matrices (determined by the function $f$ and the complexity measure) of the largest eigenvalue of the matrix.

- In Section 4, we consider the composition limit of block sensitivity. We prove Theorem 22 which says that for any boolean function $f$, the composition limit of $b s(f)$ is equal to the composition limit of $C^{*}(f)$.

- In Section 4.3, we discuss the previously mentioned example from [BSW86] and correct the analysis of $b s^{\lim }(f)$. 
- In Section 5, we give improved separations between block sensitivity and fractional block sensitivity, and between block sensitivity and certificate complexity. We present two distinct examples that give the tight lower bounds $\operatorname{crit}\left(C, C^{*}\right) \geq 2$ and $\operatorname{crit}(C, b s) \geq 2$ and an example that shows $\operatorname{crit}\left(C^{*}, b s\right) \geq 3 / 2$.

- In Appendix $\mathrm{A}$ we prove that fractional certificate complexity is within a constant factor of the randomized certificate complexity defined in Aar08.

Independently, Tal ([Tal12, Tal13]) proved results that have some overlap with our work. He showed that $b s(f)$ is not submultiplicative and proved that $\left(C^{*}\right)^{l i m}(f)=b s^{l i m}(f)$. He also observed the submultiplicativity of the measures $C(f), C^{*}(f)$, and $s(f)$. Finally, he showed a lower bound on $\operatorname{crit}\left(C, C^{*}\right)$ of $\log (26) / \log (17)$, which we improve here to the optimal constant 2 .

\section{Preliminaries}

\subsection{Combinatorial objects over an index set $I$}

Let $I$ be an arbitrary finite set, called the index set. We will be considering a large number of mathematical objects built relative to $I$.

- A map from $I$ to the nonnegative reals is called a weight function over $I$. A weight function is said to be [0,1]-valued (respectively integral, boolean) if all weights lie in [0,1] (respectively $\mathbb{Z},\{0,1\})$. A boolean weight function $w$ corresponds naturally to the subset $w^{-1}(1)$. For any weight function $w$ over $I$ and $J \subseteq I$ we write $w(J)$ for $\sum_{j \in J} w(j)$ and $|w|$ for $w(I)$.

- A weight function family over $I$ is a set of weight functions over $I$. The family is $[0,1]$-valued (respectively integral, boolean) if weight functions in the family have this property. A boolean weight function family corresponds in the obvious way to a collection of subsets (hypergraph) on $I$. We will use the terms hypergraph and boolean weight function family interchangeably.

- A boolean assignment over I or, simply, an assignment is a map from $I$ to $\{0,1\}$.

- A boolean function over $I$ is a map from assignments over $I$ to $\{0,1\}$.

We now introduce a few non-standard notions:

- A selector is a function on domain $\{0,1\}$. We typically denote selectors by vector notation $\vec{\alpha}=\left(\alpha^{0}, \alpha^{1}\right)$.

- An assignment selector is a selector $\vec{\alpha}=\left(\alpha^{0}, \alpha^{1}\right)$ where $\alpha^{0}$ and $\alpha^{1}$ are boolean assignments over $I$.

- An assignment selector $\vec{\alpha}$ is $f$-compatible for a boolean function $f$ provided that $f\left(\alpha^{0}\right)=0$ and $f\left(\alpha^{1}\right)=1$.

- A weight function selector is a selector $\vec{w}=\left(w^{0}, w^{1}\right)$ where $w^{0}$ and $w^{1}$ are weight functions over $I$. 


\subsection{Packing and covering in hypergraphs}

In the previous section we introduced both hypergraphs over $I$ and weight functions over $I$. We will also need to consider weight functions whose domain is $\mathcal{H}$ (rather than $I$ ). For a hypergraph $\mathcal{H}$ on $I$, we have the following (fairly standard) definitions:

- For a weight function $w$ on $I$, a fractional w-packing of $\mathcal{H}$ is a weight function $\lambda$ on $\mathcal{H}$ with the property that for each $i \in I$ the sum of $\lambda(E)$ over all $E$ containing $i$ is at most $w(i)$. If we omit the word fractional then $\lambda$ is assumed to be integer valued. Given $M \in \mathbb{N}$, an $M$-fold packing of $\mathcal{H}$ is an integral $w$-packing for the constant weight function $w(\cdot) \equiv M$. Thus a 1-fold packing corresponds to a collection of pairwise disjoint edges of $\mathcal{H}$, and is called simply a packing. The weight of a (fractional) packing $\lambda$, denoted $|\lambda|$ is the sum of $\lambda(E)$ over all $E \in \mathcal{H}$.

- A fractional hitting set for $\mathcal{H}$ is a weight function $\beta$ on $I$ satisfying $\beta(E) \geq 1$ for all $E \in \mathcal{H}$. If "fractional" is omitted then $\beta$ is assumed to be boolean and so corresponds to a subset $S$ of $I$ that meets every edge.

- $\nu(\mathcal{H}), \nu^{w}(\mathcal{H}), \nu^{M}(\mathcal{H})$ and $\nu^{*}(\mathcal{H})$ denote the maximum weight (size) of a packing of $\mathcal{H}$, the maximum size of a $w$-packing of $\mathcal{H}$, the maximum size of an $M$-fold packing of $\mathcal{H}$, and the maximum weight of a fractional packing of $\mathcal{H}$ respectively.

- $\tau(\mathcal{H})$ and $\tau^{*}(\mathcal{H})$ denote the size of the smallest hitting set of $\mathcal{H}$ and the minimum weight of a fractional hitting set for $\mathcal{H}$ respectively.

For a hypergraph $\mathcal{H}$ we denote by $\partial \mathcal{H}$ the hypergraph consisting of those edges of $\mathcal{H}$ that are minimal under inclusion. It is not hard to see that all of the definitions above for a hypergraph $\mathcal{H}$ only depend on $\partial \mathcal{H}$.

The following chain of relations always holds:

$$
\nu(\mathcal{H}) \leq \nu^{*}(\mathcal{H})=\tau^{*}(\mathcal{H}) \leq \tau(\mathcal{H})
$$

where the inequalities are immediate consequences of the definitions and the equality follows from the duality theorem of linear programming.

It is also known (see [SUB11, Chapter 1]) that $\tau^{*}(\mathcal{H})=\lim _{M \rightarrow \infty} \tau^{M}(\mathcal{H}) / M=\sup _{M} \tau^{M}(\mathcal{H}) / M$.

\subsection{Assemblages}

An assemblage $\mathcal{A}$ over $I$ is a map which associates each function-assignment pair $(f, x)$ to a family $\mathcal{A}_{x}(f)$ of weight functions over $I$ that is compact (when viewed as a subset of $\mathbb{R}^{I}$ ). An important special case is when all of the weight functions are $\{0,1\}$-valued, in which case $\mathcal{A}_{x}(f)$ can be viewed as a hypergraph. Some important examples of assemblages are:

- The block assemblage $\mathcal{B}$. A block of $f$ at $x$ is a subset $B$ of $I$ such that $f(x \oplus B) \neq f(x)$ where $x \oplus B$ is obtained by complementing the bits of $x$ in the positions indexed by $B$. For the block assemblage $\mathcal{B}, \mathcal{B}_{x}(f)$ is equal to the set of blocks of $f$ at $x$.

- The minblock assemblage $\partial \mathcal{B}$. A min-block of $f$ at $x$ is a block which is minimal under containment (but not necessarily minimum size). We define $\partial \mathcal{B}_{x}(f)$ to be the set of minblocks of $f$ at $x$. 
- The witness assemblage $\mathcal{W}$. A witness $w$ of $f$ at $x$ is a hitting set for $\mathcal{B}_{x}(f)$ (equivalently, for $\left.\partial \mathcal{B}_{x}(f)\right)$. For the witness assemblage $\mathcal{W}, \mathcal{W}_{x}(f)$ is the set of witnesses of $f$ at $x$. Note we view witnesses as boolean valued weight functions over the index set of $f$.

- The fractional witness assemblage $\mathcal{W}^{*}$. A fractional witness $w$ of $f$ at $x$ is a fractional hitting set for $\mathcal{B}_{x}(f)$, and $\mathcal{W}_{x}^{*}(f)$ is the set of all fractional witnesses for $f$ at $x$. Thus a weight function $w$ on $I$ belongs to $\mathcal{W}_{x}^{*}(f)$ if and only if:

$-0 \leq w(i) \leq 1$ for each $i \in I$

$-w(B) \geq 1$ for each $B \in \partial \mathcal{B}_{x}(f)$.

- The sensitivity assemblage $\Psi$. A sensitive index for $f$ at $x$ is an index $i$ such that $\{i\}$ is a block. For the sensitivity assemblage $\Psi, \Psi_{x}(f)$ consists of a single boolean weight function which is 1 on the set of indices that are sensitive for $f$ at $x$ and 0 otherwise.

\subsection{Local complexity measures}

A local complexity measure $m$ depends on a function $f$ and an input $x$ to the function. The value is written $m_{x}(f)$ and is read as the $m$-complexity of $f$ at $x$. Given such a local complexity measure we define:

$$
\begin{aligned}
m_{0}(f) & =\max \left\{m_{x}(f): x \in f^{-1}(0)\right\} \\
m_{1}(f) & =\max \left\{m_{x}(f): x \in f^{-1}(1)\right\} \\
\vec{m}(f) & =\left(m_{0}(f), m_{1}(f)\right) \\
m(f) & =\max \left\{m_{0}(f), m_{1}(f)\right\} \\
m^{\lim }(f) & =\liminf _{k \rightarrow \infty} m\left(f^{(k)}\right)^{1 / k}
\end{aligned}
$$

The measure $m(f)$ is said to be induced by a local complexity measure. Each of the following (standard) combinatorial measures of complexity of $f$, i.e., certificate complexity, fractional certificate complexity, sensitivity and block sensitivity, are induced by local complexity measures. The corresponding local measures are defined in the following subsections.

Let $m$ be induced by a local complexity measure. For a function $f$ and an $f$-compatible selector $\vec{\alpha}$, we define $\vec{m}_{\vec{\alpha}}(f):=\left(m_{\alpha^{0}}(f), m_{\alpha^{1}}(f)\right)$. Note that $\vec{m}(f) \geq \vec{m}_{\alpha}(f)$ (coordinate-wise) with equality if and only if $\alpha^{0}$ maximizes $m_{x}(f)$ over all $x \in f^{-1}(0)$ and $\alpha^{1}$ maximizes $m_{x}(f)$ over $x \in f^{-1}(1)$. In this case we say that $\vec{\alpha}$ is an m-optimal selector for $f$.

\subsection{Assemblage-based measures}

Associated to any assemblage $\mathcal{A}$ is a local complexity measure $m=m[\mathcal{A}]$ where $m_{x}(f)$ is equal to the minimum of $|w|$ over all $w \in \mathcal{A}_{x}(f)$. We say that this complexity measure is induced by assemblage $\mathcal{A}$. In this way we define the following local complexity measures:

- The certificate complexity of $f$ at $x, C_{x}(f)$ is the minimum of $|w|$ over $w \in \mathcal{W}_{x}(f)$.

- The fractional certificate complexity of $f$ at $x, C^{*}(f)$, is the minimum of $|w|$ over $w \in \mathcal{W}_{x}^{*}(f)$. 
- The sensitivity of $f$ at $x, s_{x}(f)$, is the number of sensitive indices of $f$ at $x$ which is (trivially) the size of the set in $\Psi_{x}(f)$.

Fix an assemblage $\mathcal{A}$ with associated local complexity measure $m$ and a boolean function $f$. Let $\vec{\alpha}=\left(\alpha^{0}, \alpha^{1}\right)$ be an $f$-compatible assignment selector and let $\vec{w}=\left(w^{0}, w^{1}\right)$ be a weight function selector. We say that $(\vec{\alpha}, \vec{w})$ form an $(f, \mathcal{A})$-compatible pair if $w^{0} \in \mathcal{A}_{\alpha^{0}}(f)$ and $w^{1} \in \mathcal{A}_{\alpha^{1}}(f)$. For such a compatible pair, we say $\vec{w}$ is $\vec{\alpha}$-compatible.

If $(\vec{\alpha}, \vec{w})$ form an $(f, \mathcal{A})$-compatible pair, $\left|w^{0}\right|=m_{\alpha^{0}}(f)$, and $\left|w^{1}\right|=m_{\alpha^{1}}(f)$, then we say $\vec{w}$ is an $m$-optimal selector for $f$ at $\vec{\alpha}$.

\subsection{Block sensitivity and its variants}

Next we define some local complexity measures related to packings of blocks:

- $b s_{x}(f)$, the block sensitivity of $f$ at $x$, is $\nu\left(\mathcal{B}_{x}(f)\right)$, the size of the maximum packing of blocks.

- $b s_{x}^{*}(f)$, the fractional block sensitivity of $f$ at $x$, is $\nu^{*}\left(\mathcal{B}_{x}(f)\right)$, the weight of the maximum fractional packing of blocks.

- $b s_{x}^{M}(f)$, the $M$-fold block sensitivity of $f$ at $x$, where $M$ is a positive integer, is $\nu^{M}\left(\mathcal{B}_{x}(f)\right)$, the weight of the maximum $M$-fold packing of blocks.

- $b s_{x}^{w}(f)$, the $w$-block sensitivity of $f$ at $x$, where $w$ is a weight function on $I$, is $\nu^{w}\left(\mathcal{B}_{x}(f)\right)$, the $w$-block sensitivity of $f$ at $x$.

Applying the general inequalities for hypergraph parameters (mentioned in Section 2.2) we have:

$$
s_{x}(f) \leq b s_{x}(f) \leq b s_{x}^{*}(f)=C_{x}^{*}(f) \leq C_{x}(f) .
$$

Also, we have

$$
b s^{*}(f)=\lim _{M \rightarrow \infty} b s^{M}(f) / M=\sup _{M} b s^{M}(f) / M .
$$

\subsection{Compositions}

We will need to define the composition of various objects over an index set. For this purpose, it is convenient to represent an index set as the set of leaves of a rooted tree.

We define an indexed tree to be a rooted tree $T$ with labelled edges such that for each internal node the edges to its children have distinct labels. For a node $v$, we write $C(v)=C_{T}(v)$ for the set of children of $v$ and $I(v)=I_{T}(v)$ for the set of labels on the edges from $v$ to $C(v)$. It follows that, for any node $v$, the sequence of edge labels along the path from the root to $v$ uniquely identifies $v$, and we identify $v$ we this sequence. Thus the root of the tree is the empty sequence $\Lambda$, and for each internal node $v$, the children of $v$ are nodes of the form $v s$ where $s \in I(v)$. We write $L(T)$ for the set of leaves of $T$, and $\operatorname{Int}(T)$ for the set of internal nodes (non-leaves) of $T$. The set $L(T)$ is the index set associated with $T$. In what follows we switch freely between the notion of index set and indexed tree. We also restrict attention to trees of uniform depth, that is where all leaves are at the same distance from the root.

We now define compositions of indexed trees. If $T$ is an indexed tree and $\left(T_{v}: v \in L(T)\right)$ is a family of trees indexed by $L(T)$, then the composition $T_{\circ}=T\left(T_{v}: v \in L(T)\right)$ is the indexed tree 
obtained by identifying each leaf $v$ of $T$ with the root of $T_{v}$. The index set $L\left(T_{\circ}\right)$ associated with $T_{0}$ is the set of all strings of the form $v w$ where $v$ is a leaf of $T$ and $w$ is a leaf of $T_{v}$.

Every tree $T$ can be constructed as a composition of the star from the root, with the collection of subtrees rooted at the children of the root. By applying this decomposition recursively, we can build up every tree from the collection of stars corresponding to each internal vertex.

In the special case that all $T_{v}$ are the same tree $T^{\prime}$, we say the composition is uniform and write it as $T \circ T^{\prime}$. This composition clearly forms an associative operation on indexed trees so that the notation $T_{1} \circ \cdots \circ T_{k}$ is well defined. The leaf set of $T_{1} \circ \cdots \circ T_{k}$ consists of sequences $v_{1}, \ldots, v_{k}$ where $v_{i}$ is a leaf of $T_{i}$. Such trees may be thought of as representations of product sets $I_{1} \times I_{2} \times \cdots \times I_{k}$. If all $T_{i}$ are the same tree $T$, we write this composition as $T^{(k)}$, which is the $k$-wise iterated composition of $T$.

\subsubsection{Compositions of various objects}

With the framework of indexed trees, we now define notions of compositions for various types of objects over index sets. For an appropriate object type $\tau$ the form of the composition is the same. Every object of type $\tau$ is defined with respect to an index set, and the index set is represented as the leaf set of a tree. For simplicity we say that object $\omega$ is defined over $T$ if its index set is $L(T)$.

Let $T$ be a tree, and let $\left(T_{v}: v \in L(T)\right)$ be a family of trees indexed by the leaves of $T$. We defined the composition of $T$ with $\left(T_{v}: v \in L(T)\right)$ to be the tree $T_{\circ}$ obtained by identifying the roots of each $T_{v}$ with the leaf $v$ of $T$. Recall that $L\left(T_{\circ}\right)$ consists of pairs $v w$ where $v \in L(T)$ and $w \in L\left(T_{v}\right)$.

Let $\tau$ be some type of object (such as hypergraph) over an index set. Suppose that $\omega$ is an object of type $\tau$ over the index set $L(T)$ and for each $v \in L(T)$ let $\omega_{v}$ be an object of type $\tau$ over the index set $L\left(T_{v}\right)$. For certain types $\tau$ we define a composition $\omega_{\circ}=\omega\left(\omega_{v}: v \in L(T)\right)$ over $L\left(T_{\circ}\right)$. Our composition operation for $\tau$ will combine these objects into an object $\omega_{\circ}$ over index set $L\left(T_{\circ}\right)$.

Here are compositions for some basic object types:

- Weight functions. If $\tau$ is the class of weight functions, $w$ is a weight function on $T$, and for each $v \in L(T), w_{v}$ is a weight function on $L\left(T_{v}\right)$, then the composition $w_{\circ}=w\left(w_{v}: v \in L(T)\right)$ is the weight function on $L\left(T_{\circ}\right)$ where $\omega_{\circ}(v w):=\omega(v) \omega_{v}(w)$.

- Subsets. By associating a subset of a set $I$ with the weight function given by its characteristic function, the composition of weight functions gives a notion of composition of subsets.

- Weight function families. Let $\Omega$ be a family of weight functions on $L(T)$ and for each $v \in L(T)$ let $\Omega_{v}$ be a family of weight functions over $L\left(T_{v}\right)$. Then $\Omega_{\circ}=\Omega\left(\Omega_{v}: v \in L(T)\right)$ is the weight function family on $L\left(T_{\circ}\right)$ consisting of all compositions $w\left(w_{v}: v \in L(T)\right)$ where $w \in \Omega$ and for each $v \in L(T), w_{v} \in \Omega_{v}$.

- Hypergraphs. By viewing a hypergraph as a set of boolean weight functions, the notion of composition of weight function families specializes to a notion of composition of hypergraphs.

- Boolean functions. Let $f$ be a boolean function over $L(T)$ and, for each $v \in L(T)$, let $f_{v}$ be a boolean function over $L\left(T_{v}\right)$. Then the composition $f_{\circ}=f\left(f_{v}: v \in L(T)\right)$ is the boolean function defined over $L\left(T_{\circ}\right)$ whose value on a boolean assignment over $L\left(T_{\circ}\right)$ is computed by defining $b_{v}$ for $v \in L(T)$ to be $f_{v}$ evaluated on the subset of inputs corresponding to $L\left(T_{v}\right)$ and then evaluating $f$ on assignment $\left(b_{v}: v \in L(T)\right)$. 
The notions of uniform and iterated compositions are defined in the natural way. If $T=$ $T_{1} \circ \cdots \circ T_{k}$ is a uniform composition of trees and for each $i \in[k], \Omega_{i}$ is an object of type $\tau$ over $L\left(T_{i}\right)$ then $\Omega_{1} \circ \cdots \circ \Omega_{k}$ is an object of type $\tau$ over $T$. It is easy to verify that for the various compositions we define that the operation $\circ$ is associative so that the uniform composition is welldefined. We write $\Omega^{(k)}$ for the $k$-wise iterated composition of $\Omega$.

Given an arbitrary indexed tree $T$, a $T$-ensemble of objects of type $\tau$ is an indexed family $\omega_{T}=\left(\omega_{v}: v \in \operatorname{Int}(T)\right)$ where $\omega_{v}$ is an object of type $\tau$ over the index set $I(v)$. We define the composition of $\omega_{T}$, denoted $\odot \omega_{T}$ inductively: For a null tree (consisting of only a root $\Lambda$ so that $L(T)=\{\Lambda\}$ ), there is a null object of type $\tau$. For weight functions, the null object is the weight function mapping $\Lambda$ to 1 , and for boolean functions the null object is the (univariate) identity function. For a non-null tree $T, \odot \Omega_{T}$ is given by $\Omega_{\Lambda}\left(\Omega_{v}: v \in C(\Lambda)\right)$, which is the composition of the object associated with the root with the collection of objects associated with the children of the root. Unwinding this recursion gives the following alternative description of the compositions of $T$-ensembles for various objects:

- Weight functions. Let $w_{T}=\left(w_{v}: w \in \operatorname{Int}(T)\right)$ be a $T$-ensemble of weight functions (so that $w_{v}$ is a weight function on $\left.I(v)\right)$. We can view $w_{v}$ as assigning a weight to each edge coming out of $v$. Then the composition $\odot w_{T}$ assigns a weight to each leaf $l$ which is given by the product of the weights on the edges along the path from the root to $l$.

- Subsets. By associating a subset of a set $I$ with the weight function given by its characteristic function, the composition of weight functions gives a notion of composition of subsets.

- Weight function families. Let $\Omega_{T}=\left(\Omega_{v}: v \in \operatorname{Int}(T)\right)$ be a $T$-ensemble of weight function families. Thus, for each $v, \Omega_{v}$ is a set of weight functions over $I(v)$. The composition $\odot \Omega_{T}$ is the set of all weight functions of the form $\odot w_{T}$ where $w_{T}$ is a weight function ensemble satisfying $w_{v} \in \Omega_{v}$ for each $v$.

- Hypergraphs. Let $\mathcal{H}_{T}=\left(\mathcal{H}_{v}: v \in \operatorname{Int}(T)\right\}$ be a $T$-ensemble of hypergraphs. Thus for each $v, \mathcal{H}_{v}$ is a hypergraph on $I(v)$, which can be viewed as a family of boolean-valued weight functions on $I(v)$. The composition $\odot \mathcal{H}_{T}$ is obtained by specializing the composition of weight function families, and is a hypergraph on $L(T)$

- Boolean functions. Let $f_{T}=\left(f_{v}: v \in \operatorname{Int}(T)\right)$ be a $T$-ensemble of boolean functions. The composition $\odot f_{T}$ is the function over $L(T)$ obtained by viewing $T$ as a circuit and each vertex $v$ as a gate which computes the function $f_{v}$.

- Assignment selectors. This is described more easily in the context of the next subsection, so we present it there.

\subsection{Boolean labelings of trees, and compositions of assignment selectors}

A boolean T-labeling for an indexed tree $T$ is a mapping $b_{T}=(b(v): v \in T)$ that assigns a bit to each vertex of $T$. Given a boolean $T$-labeling $b_{T}$ we define $b_{v}$, for an internal node $v$, to be the labeling $b_{T}$ restricted to the children of $v$. Note the difference between the notation $b(v)$, which is a single bit, and $b_{v}$, which is an assignment to $C(v)$. Thus, any boolean $T$-labeling $b_{T}$ induces an assignment T-ensemble $\left(b_{v}: v \in \operatorname{Int}(T)\right)$. Also, the leaf assignment determined by $b_{T}$ is the boolean assignment to the leaves obtained by restricting $b_{T}$ to $L(T)$. 
Boolean T-labelings will arise for us in two ways:

- (Bottom-up labelings) If $f_{T}=\left(f_{v}: v \in \operatorname{Int}(T)\right)$ is a $T$-ensemble of boolean functions and $\alpha \in\{0,1\}^{L(T)}$ is a boolean assignment to the leaves of $T$ then viewing $f_{T}$ as a circuit with node $v$ being a gate computing $f_{v}$, then the evaluation of $f_{T}$ on input $\alpha$, denoted $f_{T}(\alpha)$, is a boolean $T$-labeling $(b(v): v \in T)$ ) where the label $b(v)$ for a node $v$ is defined from the leaves upward as follows: if $v$ is a leaf then $b(v)=\alpha(v)$ and if $v \in \operatorname{Int}(T)$, then having defined $b(w)$ for each child $w$ of $v$ we have $b(v)=f_{v}\left(b_{v}\right)$, where as above $b_{v}$ is the assignment to $C(v)$ determined by $b$. We call the resulting boolean $T$-labeling the evaluation labeling induced by $f_{T}$ and $\alpha$.

- (Top-down labelings) If $c \in\{0,1\}$ and $\vec{\alpha}_{T}=\left(\vec{\alpha}_{v}: v \in \operatorname{Int}(T)\right)$ is a $T$-ensemble of assignment selectors, then $c$ and $\vec{\alpha}_{T}$ induce a boolean $T$-labeling in the following way. Label the root by $b(r)=c$. Now starting from the root apply the following procedure: Having labeled an internal node $v$ by $b(v)$, use the bit $b(v)$ to select the assignment $\alpha_{v}^{b(v)}$ from the assignment selector $\vec{\alpha}_{v}$, and then label the children of $v$ according to $\alpha_{v}^{b(v)}$. We call this the boolean $T$ labeling induced by root label $c$ and $\vec{\alpha}_{T}$. Note that by definition the assignment $T$-ensemble associated to $b,\left(b_{v}: v \in \operatorname{Int}(T)\right)$ is given by $b_{v}=\alpha_{v}^{b(v)}$.

If we don't specify a bit $c$, then the $T$-ensemble of assignment selectors $\vec{\alpha}_{T}$ defines a boolean $T$-labeling selector $\vec{b}_{T}=\left(b_{T}^{0}, b_{T}^{1}\right)$, where for $c \in\{0,1\}, b_{T}^{c}$ is the boolean $T$-labeling induced by $c$ and $\vec{\alpha}_{T}$. We call this the boolean $T$-labeling selector induced by $\vec{\alpha}_{T}$.

The top-down construction implicitly provides a natural notion of composition of assignment selectors:

Composition of assignment selectors. Let $\vec{\alpha}_{T}=\left(\vec{\alpha}_{v}: v \in \operatorname{Int}(T)\right)$ be a T-ensemble of assignment selectors. Let $\vec{b}_{T}$ be the boolean $T$-labeling selector induced by $\vec{\alpha}_{T}$. If we restrict each of the labelings $\vec{b}_{T}^{1}$ and $\vec{b}_{T}^{0}$ to $L(T)$ we get an assignment selector over $L(T)$. This assignment selector is defined to be the composition of the ensemble $\vec{\alpha}_{T}$ and is denoted $\vec{\alpha}_{\circ}=\left(\alpha_{\circ}^{0}, \alpha_{\circ}^{1}\right)=\odot \vec{\alpha}_{T}$.

As with compositions of other objects, we specialize composition of assignment selectors to the case of uniform compositions, and denote a uniform composition of assignment selectors by $\vec{\alpha}_{1} \circ \cdots \circ \vec{\alpha}_{k}$.

Observe that the bottom-up labelings and top-down labelings fit together in the following way. Suppose $f_{T}$ is a $T$-ensemble of boolean functions and $\vec{\alpha}_{T}$ is a $T$-ensemble of assignment selectors. Suppose that for each vertex $v \in \operatorname{Int}(T), \vec{\alpha}_{v}$ is $f_{v}$-compatible, which we defined earlier to mean $f_{v}\left(\alpha_{v}^{0}\right)=0$ and $f_{v}\left(\alpha_{v}^{1}\right)=1$. In this case we say that the ensemble $\vec{\alpha}_{T}$ is $f_{T^{-}}$-compatible.

Proposition 4. Let $f_{T}$ be a T-ensemble of boolean functions and $\vec{\alpha}_{T}$ be a T-ensemble of assignment selectors. Let $\vec{b}_{T}$ be the boolean T-labeling selector induced (top-down) by $\vec{\alpha}_{T}$ and let $\vec{\alpha}_{\circ}$ be the composition $\odot \vec{\alpha}_{T}$ (which was defined to be the restriction of $\vec{b}_{T}$ to $L(T)$ ). If for each $v \in \operatorname{Int}(T)$, $\vec{\alpha}_{v}$ is $f_{v}$ compatible with $f_{v}$ then:

- For $c \in\{0,1\}$, The (bottom-up) labeling induced by $f_{T}$ and $\alpha_{\circ}^{c}$ is $b_{T}^{c}$.

- The composed assignment selector $\odot \vec{\alpha}_{T}$ is $F$-compatible, where $F=\odot f_{T}$ is the composition of $f_{T}$. 
Proof. For $c \in\{0,1\}$, let $a_{T}^{c}$ be the labeling induced by $f_{T}$ and $\alpha_{\circ}^{c}$. We prove that, for all $v \in T$, $a_{T}^{c}(v)=b_{T}^{c}(v)$. We proceed by induction on the size of the subtree rooted at $v$. For $v \in L(T)$ we have $a_{T}^{c}(v)=\alpha_{\circ}^{c}(v)=b_{T}^{c}(v)$. For $v \in \operatorname{Int}(T)$, by the induction hypothesis, we have $a_{T}^{c}(w)=b_{T}^{c}(w)$ for all children $w$ of $v$, equivalently, we have $a_{v}=b_{v}$. Now by the definition of $a_{T}$ we have $a(v)=f_{v}\left(a_{v}\right)=f_{v}\left(b_{v}\right)$. On the other hand, by the definition of $b_{T}$ we have that $b_{v}$ is equal to $\alpha_{v}^{b(v)}$, and since $\vec{\alpha}_{v}$ is compatible with $f_{v}$ this implies $f\left(b_{v}\right)=b(v)$ and so $b(v)=a(v)$, as required.

For the second part, the composed assignment $\vec{\alpha}_{\circ}$ is (by definition) equal to $\vec{b}_{T}$ restricted to $L(T)$, and by the first part this is the same as $\vec{a}_{T}$ restricted to $L(T)$. For each $c \in\{0,1\}$, the value of $f_{T}$ at $a_{T}^{c}$ is the value of the root in the bottom-up labeling (viewing $T$ as a circuit with gates $\left.\left(f_{v}: v \in \operatorname{Int}(T)\right)\right)$, and this is the label given to the root by $a_{T}^{c}=b_{T}^{c}$ which is equal to $c$ by the definition of the top-down labeling $b_{T}^{c}$

\section{The growth of various complexity measures under iterated com- position}

Our goal in this section is to understand how $m\left(f^{(k)}\right)$ relates to $m(f)$ for various complexity measures. In Section 3.1 we provide a high level discussion of how to analyse $m\left(f^{(k)}\right)$. To do so we will initially state, without proofs, the lemmas which lead to the main result. We will then prove the main theorem modulo these lemmas. Finally, in Section [3.2, we will provide all the remaining proofs and definitions which were left out.

\subsection{Analysing $m\left(f^{k}\right)$}

Fix an assemblage $\mathcal{A}$ and let $m$ be the associated complexity measure. Informally, $m(f)$ is high if there is a hard input $\alpha$, which means that every weight function in $\mathcal{A}_{\alpha}(f)$ has high total weight.

Let's start with the most general function composition, where $F$ is the composition of a $T$ ensemble $\left.f_{T}=\left(f_{v}: v \in \operatorname{Int}(T)\right\}\right)$ for an arbitrary tree $T$ of uniform depth. Fix an input $\alpha$ to the leaves of $T$ and let $(b(v): v \in T)$ be the evaluation labeling of $f_{T}$ on $\alpha$ and $\left(b_{v}: v \in \operatorname{Int}(T)\right)$ be the corresponding assignment ensemble. Recall that, for each $v \in \operatorname{Int}(T)$, we have $b(v)=f_{v}\left(b_{v}\right)$.

To determine $m_{\alpha}(F)$ we want to determine the minimum weight of a weight function $w \in \mathcal{A}_{\alpha}(F)$. In trying to analyze this minimum, it is natural to look at weight functions which are representable as $T$-compositions of weight functions as follows: For each internal node $v$ of $T$ select a weight function $w_{v}$ that belongs to the set of weight functions $\mathcal{A}_{b_{v}}\left(f_{v}\right)$, and take $w$ to be the composition of the $T$-ensemble $\left(w_{v}: v \in \operatorname{Int}(T)\right)$. We say that such an ensemble is compatible with $\left(f_{v}: v \in \operatorname{Int}(T)\right)$ and $\alpha$. Our hope is that the minimum weight of a weight function in $\mathcal{A}_{\alpha}(F)$ is attained by such a composition. It is easy to show that this is true provided that the assemblage $\mathcal{A}$ satisfies the following two properties:

1. For any weight function ensemble that is compatible with $\left(f_{v}: v \in \operatorname{Int}(T)\right)$ and an assignment $\alpha$, its composition belongs $\mathcal{A}_{\alpha}(F)$.

2. If $w$ is any weight function in $\mathcal{A}_{\alpha}(F)$ then there is a weight function ensemble $\left(w_{v}: v \in \operatorname{Int}(T)\right)$ that is compatible with $\left(f_{v}: v \in \operatorname{Int}(T)\right)$ and $\alpha$ whose composition has total weight less than that of $w$. 
We call an assemblage well behaved if it satisfies (1) and (2). Summarizing the above, we have:

Proposition 5. Let $m$ be a complexity measure associated to a well-behaved assemblage. Let $\left(f_{v}: v \in \operatorname{Int}(T)\right)$ be a T-ensemble of functions with composition $F$ and let $\alpha$ be an input to $F$. Then

$$
m_{\alpha}(F)=\min |w|,
$$

where $w$ ranges over all compositions of weight function ensembles $\left(w_{v}: v \in \operatorname{Int}(T)\right)$ that are compatible with $\left(f_{v}: v \in \operatorname{Int}(T)\right)$ and $\alpha$.

In section 3.2, we will prove

Lemma 6. Each of the assemblages $\partial \mathcal{B}, \mathcal{W}, \mathcal{W}^{*}$ and $\Psi$ are well-behaved.

This implies that Proposition [5 can be applied to certificate complexity, fractional certificate complexity and sensitivity. In the remaining discussion we assume that the assemblage $\mathcal{A}$ is wellbehaved.

At this point, we restrict attention to $F$ which are uniform compositions $F=f_{1} \circ \cdots \circ f_{k}$ where $f_{i}$ is a boolean function over the index set $L\left(T_{i}\right)$ where $T_{i}$ is an indexed star. To understand $m(F)$ we want to identify an input $\alpha$ that maximizes $m_{\alpha}(F)$. Actually we'll try to identify an assignment selector $\vec{\alpha}=\left(\alpha^{0}, \alpha^{1}\right)$ for $F$ that is $m$-optimal for $F$, which (as defined in Section 2.4) means that $\alpha^{0}$ maximizes $m_{\alpha}(F)$ over $\alpha \in F^{-1}(0)$ and $\alpha^{1}$ maximizes $m_{\alpha}(F)$ over $\alpha \in F^{-1}(1)$. It is natural to speculate that we can obtain such an assignment selector $\vec{\alpha}$ as a composition of assignment selectors $\vec{\alpha}_{1} \circ \cdots \circ \vec{\alpha}_{k}$ where $\vec{\alpha}_{i}$ is an assignment selector for $f_{i}$. This indeed turns out to be the case, as is stated in the second part of the following lemma:

Lemma 7. Let $f_{1}, \ldots, f_{k}$ be a sequence of boolean functions and let $F$ be their composition.

- If $\vec{\alpha}_{1}, \ldots, \vec{\alpha}_{k}$ are assignment selectors, where $\vec{\alpha}_{i}$ is $f_{i}$-compatible, then $\vec{\alpha}:=\vec{\alpha}_{1} \circ \cdots \circ \vec{\alpha}_{k}$ is an F-compatible assignment selector.

- There are assignment selectors $\vec{\alpha}_{1}, \ldots, \overrightarrow{\alpha_{k}}$, where $\vec{\alpha}_{i}$ is $f_{i}$-compatible, such that $\vec{\alpha}:=\overrightarrow{\alpha_{1}} \circ \cdots \circ \overrightarrow{\alpha_{k}}$ is an m-optimal selector for $F$.

Note that it is not the case in this lemma that each $\vec{\alpha}_{i}$ in the conclusion is $m$-optimal for $f_{i}$. Nevertheless, the lemma is useful because, in evaluating $m(F)$, it is enough to consider all assignment selectors of the form $\vec{\alpha}_{1} \circ \cdots \circ \vec{\alpha}_{k}$ where each $\alpha_{i}$ is $f_{i}$-compatible.

Now consider such a composed assignment selector $\vec{\alpha}$. We want to understand $m_{\alpha^{0}}(F)$ and $m_{\alpha^{1}}(F)$ and for this it suffices to determine the weight functions $w^{0} \in \mathcal{A}_{\alpha^{0}}(F)$ and $w^{1} \in \mathcal{A}_{\alpha^{1}}(F)$ of minimum weight. One might hope that $w^{0}$ and $w^{1}$ can each be expressed as a uniform composition of weight functions, but this need not be true. Once again we need to consider compositions of weight function selectors rather than weight functions. There is a natural way to compose any sequence of weight function selectors, but it is a bit complicated because it depends not only on the sequence $\vec{w}_{1}, \ldots, \vec{w}_{k}$ but also on the associated assignment selectors $\vec{\alpha}_{1}, \ldots, \vec{\alpha}_{k}$. What we end up with is a composition operation which acts on pairs $\left(\vec{\alpha}_{i}, \vec{w}_{i}\right)$ consisting of an assignment selector and weight function selector for $f_{i}$ and produces such a pair $(\vec{\alpha}, \vec{w})$ for $f$. We call this an assignmentweight selector-pair, or simply $A W$-selector pair. The assignment selector $\vec{\alpha}$ is just the composition of the assignment selectors $\vec{\alpha}_{i}$ as before (and does not depend on the $\vec{w}_{i}$, but $\vec{w}$ depends on both the 
$\vec{\alpha}_{i}$ and $\vec{w}_{i}$ ). Again, due to the technical nature of this construction, we delay the explicit definition for Section 3.2. For now it is enough to note that this composition operation satisfies the following properties (see Section 2.5 for the definition of $(f, \mathcal{A})$-compatible):

Lemma 8. Let $f_{1}, \ldots, f_{k}$ be a sequence of boolean functions and let $F$ be their composition. Let $\vec{\alpha}_{1}, \ldots, \vec{\alpha}_{k}$ be assignment selectors such that each $\vec{\alpha}_{i}$ is $f_{i}$-compatible. Then the following hold:

- For any sequence $\vec{w}_{1}, \ldots, \vec{w}_{k}$ of weight function selectors, where $\left(\vec{\alpha}_{i}, \vec{w}_{i}\right)$ is $\left(f_{i}, \mathcal{A}\right)$ compatible, the composition $(\vec{\alpha}, \vec{w}):=\left(\vec{\alpha}_{1}, \vec{w}_{1}\right) \circ \cdots \circ\left(\vec{\alpha}_{k}, \vec{w}_{k}\right)$ is $(F, \mathcal{A})$-compatible.

- There are weight function selectors $\vec{w}_{1}, \cdots, \vec{w}_{k}$ such that the weight function $\vec{w}$ that comes from the composition $(\vec{\alpha}, \vec{w}):=\left(\vec{\alpha}_{1}, \vec{w}_{1}\right) \circ \cdots \circ\left(\vec{\alpha}_{k}, \vec{w}_{k}\right)$ is an m-optimal weight function selector for $F$ at $\vec{\alpha}$.

We now define the following function, which maps a sequence of AW selector-pairs to a real number:

$$
V\left(\left(\vec{\alpha}_{1}, \vec{w}_{1}\right), \ldots,\left(\vec{\alpha}_{k}, \vec{w}_{k}\right)\right):=\max \left\{\left|w^{0}\right|,\left|w^{1}\right|\right\},
$$

where the weight function selector $\vec{w}$ is given by $(\vec{\alpha}, \vec{w})=\left(\vec{\alpha}_{1}, \vec{w}_{1}\right) \circ \cdots \circ\left(\vec{\alpha}_{k}, \vec{w}_{k}\right)$. Combining Lemmas 7 and 8 we obtain

Lemma 9. Let $F=f_{1} \circ \cdots \circ f_{k}$. Then $m(F)$ is equal to the maximum, over all sequences of assignment selectors $\vec{\alpha}_{1}, \ldots, \vec{\alpha}_{k}$ where each $\vec{\alpha}_{i}$ is $f_{i}$-compatible, of the minimum, over all sequences $\vec{w}_{1}, \ldots, \vec{w}_{k}$ of weight function selectors such that $\left(\vec{\alpha}_{i}, \vec{w}_{i}\right)$ is $\left(f_{i}, \mathcal{A}\right)$-compatible, of $V\left(\left(\vec{\alpha}_{1}, \vec{w}_{1}\right), \ldots,\left(\vec{\alpha}_{k}, \vec{w}_{k}\right)\right)$.

So next we want to understand the function $V$. The following definitions will be helpful.

- Largest eigenvalue. For a square real matrix $A, \rho(A)$ denotes the maximum of $|\lambda|$ over all eigenvalues $\lambda$ of $A$.

- The profile matrix of an AW selector-pair $(\vec{\alpha}, \vec{w})$ is defined to be the 2 by 2 matrix $M_{\vec{\alpha}, \vec{w}}$ with rows and columns indexed by $\{0,1\}$ with $s, t$ entry equal to $\sum_{j} w^{s}(j)$ where the sum ranges over indices $j$ such that $\alpha_{j}^{s}=t$. Note that for $s \in\{0,1\}$, the $s$ th row sum of $M_{\vec{\alpha}, \vec{w}}$ is equal to $\left|w^{s}\right|$.

- Profile matrix family $\mathcal{M}_{\vec{\alpha}}(f)$ for the assemblage $\mathcal{A}$. For each function $f$ and assignment selector $\vec{\alpha}, \mathcal{M}_{\vec{\alpha}}(f)$ is the set of all matrices $M_{\vec{\alpha}, \vec{w}}$ where $\vec{w}$ ranges over weight function selectors such that $(\vec{\alpha}, \vec{w})$ form an $(f, \mathcal{A})$-compatible pair. Note that the set of profile matrices depends on the assemblage. In particular, the set of profile matrices for the assemblage $\mathcal{W}$ will be a subset of the set of profile matrices for the assemblage $\sqsupseteq *$.

Example. Consider $f=\mathrm{NAND}_{n}(x)$, that is $f(x)=0$ only at the all 1 's input. Let $\alpha^{0}=$ $(1,1, \cdots, 1)$ and $\alpha^{1}=(0,1,1,1, \cdots, 1)$. Take $m$ to be certificate complexity. The only witness for $\alpha^{0}$ is $w_{0} \equiv 1$, and we take $w_{1}$ to assign weight 1 on the 0 index and weight 0 otherwise. Here the profile matrix

$$
M_{\vec{\alpha}, \vec{w}}=\left[\begin{array}{cc}
0 & n \\
1 & 0
\end{array}\right] .
$$


Note that $\rho\left(M_{\vec{\alpha}, \vec{w}}\right)=\sqrt{n}$. It is also true that $C^{\lim }(f)=\sqrt{n}$ (this is not a coincidence as we will see).

It turns out that matrix multiplication captures the mechanism behind the composition of AW selector-pairs. In fact, for any sequence $\left\{\left(\vec{\alpha}_{i}, \vec{w}_{i}\right)\right\}_{i=1}^{k}$ we have that $V\left(\left(\vec{\alpha}_{1}, \vec{w}_{1}\right), \ldots,\left(\vec{\alpha}_{k}, \vec{w}_{k}\right)\right)$ is equal to the maximum row sum of the product $M_{\vec{\alpha}_{1}, \vec{w}_{1}} \cdots M_{\vec{\alpha}_{k}, \vec{w}_{k}}$. This follows from

Proposition 10. For any sequence $\left(\vec{\alpha}_{1}, \vec{w}_{1}\right), \ldots,\left(\vec{\alpha}_{k}, \vec{w}_{k}\right)$ of AW selector-pairs, if $(\vec{\alpha}, \vec{w})$ is their composition then the profile matrix $M_{\vec{\alpha}, \vec{w}}$ is given by:

$$
M_{\vec{\alpha}, \vec{w}}=M_{\vec{\alpha}_{1}, \vec{w}_{1}} \cdots M_{\vec{\alpha}_{k}, \vec{w}_{k}} .
$$

Lemma 9 and Proposition 10 imply

Corollary 11. Let $F=f_{1} \circ \cdots \circ f_{k}$. Then $m(F)$ is equal to the maximum, over all sequences $\vec{\alpha}_{1}, \ldots, \vec{\alpha}_{k}$ where $\vec{\alpha}_{i}$ is $f_{i}$-compatible, of the minimum, over all choices of matrices $M_{1}, \ldots, M_{k}$ where $M_{i}$ belongs to the profile matrix family $\mathcal{M}_{\vec{\alpha}}\left(f_{i}\right)$, of the maximum row sum of the product $M_{1} \cdots M_{k}$.

At last we are ready to consider the case of iterated composition, where all of the $f_{i}$ are the same function $f$. We wish to understand $m\left(f^{(k)}\right)$, which we now know is the maximum over choices of $\vec{\alpha}_{i}$ of the minimum over choices of $\vec{w}_{i} \in \mathcal{A}_{\vec{\alpha}_{i}}(f)$ of $V\left(\left(\vec{\alpha}_{1}, \vec{w}_{1}\right) \circ \cdots \circ\left(\vec{\alpha}_{k}, \vec{w}_{k}\right)\right)$. Intuitively, one may think of the choice of each assignment selector $\vec{\alpha}_{i}$ as defining the set of profile matrices $\mathcal{M}_{\vec{\alpha}_{i}}(f)$, and the choice of each weight function selector $\vec{w}_{i}$ as choosing a matrix $M_{i} \in \mathcal{M}_{\vec{\alpha}_{i}}(f)$.

If we wish to lower bound $m\left(f^{(k)}\right)$ we hope to find assignment selectors $\vec{\alpha}_{i}$ for which all possible products of the form

$$
M_{1} M_{2} \cdots M_{k}, \quad M_{i} \in \mathcal{M}_{\vec{\alpha}_{i}}(f)
$$

have a large max row sum. We will need the following simple fact about matrices:

Fact 12. For any matrix $M \in \mathbb{R}_{\geq 0}^{2 \times 2}$ we have

$$
\|M\|_{\infty} \geq \rho(M) / 2 .
$$

It turns out that the minimum of $\rho(M)$ over matrices in the family $\mathcal{M}_{\vec{\alpha}}(f)$ gives a good notion of how hard the input selector $\vec{\alpha}$ is. We now introduce two more definitions:

- The characteristic value $\hat{m}_{\vec{\alpha}}(f)$ for $(f, \vec{\alpha})$. For a boolean function $f$ and assignment selector $\vec{\alpha}$ compatible with $f$, define $\hat{m}_{\vec{\alpha}}(f)$ to be the minimum of $\rho(A)$ over all $A \in \mathcal{M}_{\vec{\alpha}}(f)$. The function $\hat{m}_{\vec{\alpha}}(f)$ can be viewed as another complexity measure derived from the assemblage $\mathcal{A}$ which is bi-local rather than local in the sense that it depends on a pair of assignments rather than just one.

- The characteristic value $\hat{m}(f)$ is the maximum, over all assignment selectors $\vec{\alpha}$ compatible with $f$, of $\hat{m}_{\vec{\alpha}}(f)$.

If we can prove that $m_{\vec{\alpha}}(f) \geq \lambda$ then this will imply by Fact 12 that $\max \left(m_{\alpha_{0}}(f), m_{\alpha_{1}}(f)\right) \geq \lambda / 2$. This suggests that, in order to construct a hard assignment selector for $f^{(k)}$, we should choose a selector $\vec{\beta}$ which maximizes $\hat{m}_{\vec{\beta}}(f)$ and then set $\vec{\alpha}=\vec{\beta}^{(k)}$. We will use this idea to prove 
Lemma 13. For any boolean function $f$ and $k \in \mathbb{N}$

$$
m\left(f^{(k)}\right) \geq \hat{m}(f)^{k} / 2 .
$$

Next we obtain an upper bound $m\left(f^{(k)}\right)$. We show that for any sequence of assignment selectors $\vec{\alpha}_{1}, \cdots, \vec{\alpha}_{k}$, we can find matrices $M_{i} \in \mathcal{M}_{\alpha_{i}}(f)$ such that the product $M=M_{1} M_{2} \cdots M_{k}$ has no entry larger than $n k(\hat{m}(f))^{k-1}$. This will prove

Lemma 14. For any boolean function $f$ on $n$ variables and $k \in \mathbb{N}$,

$$
m\left(f^{(k)}\right) \leq 2 n k(\hat{m}(f))^{k-1} .
$$

Armed with these lemmas, we easily obtain the main result of this section:

Theorem 15. Let $m$ be a complexity measure with associated well-behaved assemblage $\mathcal{A}$. Then, for any boolean function $f, m^{\lim }(f)=\hat{m}(f)$.

Proof. Assume $f$ is a function on $n$ variables. Recall that $m^{\lim }(f):=\lim _{k \rightarrow \infty} m\left(f^{(k)}\right)^{1 / k}$. By Lemmas 13 and 14 we have that

$$
\lim _{k \rightarrow \infty}(1 / 2)^{1 / k}(\hat{m}(f)) \leq m^{\lim }(f) \leq \lim _{k \rightarrow \infty}(2 n k)^{1 / k} \hat{m}(f) .
$$

Both the above limits approach $\hat{m}(f)$, thus the result follows.

This concludes the informal discussion of the main result. We note that Theorem 15 has been proven modulo all lemmas and propositions stated in this section. It remains to explicitly define the composition of assignment selectors, and AW selector-pairs, and provide the proofs which were left out of this section. All such proofs and definitions are in the following section.

\subsection{Filling in the details}

In this section we prove the technical lemmas which were referred to in the previous section.

Our first step is to prove Lemma 6 that sensitivity, certificate complexity, and fractional certificate complexity all induced by well behaved assemblages. The following proposition shows that certificates (respectively fractional certificates) compose and decompose nicely.

Proposition A. Let $T$ be an indexed tree, and let $\left(\Omega_{v}: v \in \operatorname{Int}(T)\right)$ be an ensemble of boolean valued weight function families (i.e., hypergraphs). Let $\Omega_{T}$ be the composition $\odot_{T}\left(\Omega_{v}: v \in \operatorname{Int}(T)\right)$.

- If $\left(h_{v}: v \in \operatorname{Int}(T)\right)$ is a T-ensemble of weight functions such that each $h_{v}$ is a fractional hitting set for $\Omega_{v}$, then $h_{T}=\odot_{T}\left(h_{v}: v \in \operatorname{Int}(T)\right)$ is a fractional hitting set for $\Omega_{T}$. Furthermore, if all of the $h_{v}$ are boolean valued (so that $h_{v}$ is a hitting set), then so is $h_{T}$.

- If $h$ is any fractional hitting set for $\Omega_{T}$, then there exists a T-ensemble of weight functions $\left(h_{v}: v \in \operatorname{Int}(T)\right)$ such that each $h_{v}$ is a hitting set for $\Omega_{v}$, and $h \geq_{T} \odot_{T}\left(h_{v}: v \in \operatorname{Int}(T)\right)$ pointwise. Furthermore, if $h$ is boolean valued, then all of the $h_{v}$ can be chosen to be boolean valued. 
Proof. For both parts of the lemma we first prove the case where every leaf in $T$ is distance 2 from the root, and then use induction to obtain the general result.

Recall that $h_{T}$ assigns to leaf $l$ the product of the values that $h_{v}$ assigns to the edges along the unique path from the root to $l$. Thus, if all the $h_{v}$ are boolean valued, then $h_{T}$ is boolean valued. We now show that, for all $w \in \Omega_{T}$,

$$
\sum_{l \in L(T)} h_{T}(l) w(l) \geq 1
$$

Let $r$ be the root of $T$ and fix $w \in \Omega_{T}$. Since $\Omega_{T}=\Omega_{r}\left(\Omega_{v}: v \in C(r)\right)$, it follows that for some choice of $w_{r} \in \Omega_{r}$ and $w_{v} \in \Omega_{v}$ (for each $v \in C(r)$ ) we have

$$
w=w_{r}\left(w_{v}: v \in C(r)\right) \text {. }
$$

Likewise, by assumption

$$
h_{T}=h_{r}\left(h_{v}: v \in C(r)\right) .
$$

For $v \in C(r)$, let $T_{v}$ be the subtree whose root is $v$. It follows that

$$
\begin{aligned}
\sum_{l \in L(T)} h_{T}(l) w(l) & =\sum_{v \in C(r)} \sum_{l \in L\left(T_{v}\right)} h_{r}(v) h_{v}(l) w_{r}(v) w_{v}(l) \\
& =\sum_{v \in C(r)} h_{r}(v) w_{r}(v)\left(\sum_{l \in L\left(T_{v}\right)} h_{v}(l) w_{v}(l)\right) .
\end{aligned}
$$

Each $h_{v}$ is a fractional hitting set for $\Omega_{v}$, thus the inner sums are all at least 1 . Therefore, the above is

$$
\geq \sum_{v \in C(r)} h_{r}(v) w_{r}(v)
$$

This, however, is at least 1 because $h_{r}$ is a fractional hitting set for the hypergraph $\Omega_{r}$. This proves (11).

To see the induction step, for view an arbitrary tree $T$ of uniform depth as a composition $T_{r}\left(T_{v}: v \in C(r)\right)$ where $T_{r}$ is a rooted star. Then let $h_{v}=\odot_{T_{v}}\left(h_{u}: u \in \operatorname{Int}\left(T_{v}\right)\right)$, which by induction will be a fractional hitting set for $\Omega_{v}:=\odot_{T_{v}}\left(\Omega_{u}: u \in \operatorname{Int}\left(T_{v}\right)\right)$. We may then ignore the inner structure of the subtrees $T_{v}$, treating them as rooted stars, which reduces the problem to the depth 2 case already shown.

For the next part we show that, given any $h_{T}$ which is a hitting set for $\Omega_{T}$, we can find weight functions $h_{r}$ and $\left\{h_{v}: v \in C(r)\right\}$ such that $h_{T} \geq h_{r}\left(h_{v}: v \in C(r)\right)$ pointwise and all the $h_{v}$, and $h_{r}$ are hitting sets for $\Omega_{v}$ and $\Omega_{r}$ respectively.

The construction is as follows: For each $v \in C(r)$ we define

$$
h_{r}(v):=\min \left(1, \min _{w \in \Omega_{v}} \sum_{l \in L\left(T_{v}\right)} w(l) h_{T}(l)\right) .
$$

Note that $h_{r}(v)$ will be boolean valued if $w$ and $h_{T}$ are. Let $S=\left\{v \in C(r): h_{r}(v) \neq 0\right\}$. For $v \in S$ and $l \in L\left(T_{v}\right)$ we define $h_{v}(l):=h_{T}(l) / h_{r}(v)$. For $v \notin S$, we set $h_{v} \equiv 1$. Again, each $h_{v}$ 
defined in this way will be boolean valued if $h_{T}$ is boolean valued. It is clear by construction that $h_{T} \geq h_{r}\left(h_{v}: v \in \operatorname{Int}(T)\right)$.

By construction, each $h_{v}$ is a hitting set for $\Omega_{v}$. This is trivial if $v \notin S$. Otherwise, if $v \in S$ and $w \in \Omega_{v}$, then

$$
\begin{aligned}
\sum_{l \in L\left(T_{v}\right)} w(l) h_{v}(l) & =\sum_{l \in L\left(T_{v}\right)} \frac{w(l) h_{T}(l)}{h_{r}(v)} \\
& \geq \frac{\sum_{l \in L\left(T_{v}\right)} w(l) h_{T}(l)}{\min _{v} h_{r}(v)} \geq \frac{\sum_{l \in L\left(T_{v}\right)} w(l) h_{T}(l)}{\min _{w^{\prime} \in \Omega_{v}} \sum_{l \in L\left(T_{v}\right)} w^{\prime}(l) h_{T}(l)} \\
& \geq 1 .
\end{aligned}
$$

It remains to show that $h_{r}$ is a hitting set for $\Omega_{r}$. Let $w_{r} \in \Omega_{r}$ be given. For each $v \in C(r)$, let $w_{v} \in \Omega_{v}$ be such that $\min \left(1, \sum_{l \in L\left(T_{v}\right)} w_{v}(l) h_{T}(l)\right)=h_{r}(v)$. Define $w_{T}:=w_{r}\left(w_{v}: v \in C(r)\right)$. Note that $w_{T} \in \Omega_{T}$ because $\Omega_{T}=\Omega_{r}\left(\Omega_{v}: v \in C(r)\right)$. In the following analysis recall that $w_{r}$ is boolean valued by assumption.

$$
\begin{aligned}
\sum_{v \in C(r)} w_{r}(v) h_{r}(v) & =\sum_{v \in C(r)} w_{r}(v) \min \left(1, \sum_{l \in L\left(T_{v}\right)} w_{v}(l) h_{T}(l)\right) \\
& =\sum_{v \in C(r): w_{r}(v)=1} \min \left(1, \sum_{l \in L\left(T_{v}\right)} w_{v}(l) h_{T}(l)\right) \\
& \geq \min \left(1, \sum_{v \in C(r): w_{r}(v)=1} \sum_{l \in L\left(T_{v}\right)} w_{v}(l) h_{T}(l)\right) \\
& \geq \min \left(1, \sum_{l \in L(T)} w_{T}(l) h_{T}(l)\right) \\
& \geq 1 .
\end{aligned}
$$

The induction step works as follows. Given a tree $T$ of uniform depth $k$, view it as a composition $T_{r}\left(T_{v}: v \in C(r)\right)$ where each $T_{v}$ has depth $k-1$. Then decompose $\Omega_{T}=\Omega_{r}\left(\Omega_{T_{v}}: v \in C(r)\right)$, where each $\Omega_{T_{v}}$ is the $T_{v}$-composition of $\left(\Omega_{u}: u \in \operatorname{Int}\left(T_{v}\right)\right)$. Apply the height 2 case to get $h_{r}$ and $h_{T_{v}}$ with the desired properties. Then continue this process on each of the subtrees $T_{v}$ until $h_{T}$ has been fully decomposed.

We are now ready to prove Lemma 6, which we repeat for convenience.

Lemma 6. Each of the assemblages $\partial \mathcal{B}, \mathcal{W}, \mathcal{W}^{*}$ and $\Psi$ are well-behaved.

Proof. We prove each part separately. To prove that the minblock assemblage is well behaved, we only prove the case where $T$ is an indexed tree of height 2 , that is where $F=f_{r}\left(f_{v}: v \in C(r)\right)$ is 
a composition of boolean functions and $T=T_{r}\left(T_{v}: v \in C(r)\right)$. The general case will then follow by induction (we omit this part as it follows similarly to the induction in Proposition A).

The assemblage $\partial \mathcal{B}$ is well-behaved:

We will prove the stronger statement:

Claim B. For each input $x$ to $F$,

$$
\partial \mathcal{B}_{x}(F)=\odot_{T}\left(\partial \mathcal{B}_{x_{v}}\left(f_{v}\right)\right)
$$

Let $x$ be an arbitrary input for $F$. Let $\left(x_{v}: v \in \operatorname{Int}(T)\right)$ be the assignment ensemble induced by evaluating the circuit for $F$ on assignment $x$. Let $B_{T}$ be a min-block for $F$ at $x$. $B_{T}$ induces a boolean valued weight function over $C(r)$ in the following natural way: $B_{r}(v):=1$ if and only if there exists a leaf $l \in L\left(T_{v}\right)$ such that $B_{T}(l)=1$. Likewise, $B_{T}$ induces weight functions $B_{v}$ on the leaves of the subtrees rooted at $v$ for $v \in C(r)$ in the same way, that is $B_{v}(l):=1$ if and only if $B_{T}(l)=1$. In this way, $B_{T}=B_{r}\left(B_{v}: v \in C(r)\right)$. It remains to show that each $B_{v}$ which is not identically 0 is a min block for $f_{v}$ at $x_{v}$ and that $B_{r}$ is a min block for $f_{r}$ at $x_{r}$.

First we show that, for each $v \in C(r), B_{v}$ is a block at $x_{v}$. Suppose for contradiction that $f_{v}\left(x_{v} \oplus B_{v}\right)=f_{v}\left(x_{v}\right)$ for some $v \in C(r)$ where $B_{v}$ is not empty. Then changing $B_{T}$ to be 0 on the leaves of $v$ will create a strictly smaller block $B_{T}^{\prime}$. Similarly, if $B_{v}$ is a block but not a min-block, then again $B_{T}$ may be modified to be strictly smaller. Thus each $B_{v}$ is a min block for $f_{v}$ for $v \in C(r)$.

Now we show that $B_{r}$ is a min block. Recall that we defined $B_{r}(v)=1$ if and only if $B_{v}$ is not identically 0 . Furthermore, we just showed that if $B_{v}$ is not identically 0 , then it is a min block. Therefore, it follows that $B_{r}(v)=1$ if and only if $f_{v}\left(x_{v} \oplus B_{v}\right) \neq f_{v}\left(x_{v}\right)$. This implies that $F\left(x \oplus B_{T}\right)=f_{r}\left(x_{r} \oplus B_{r}\right)$. Thus, since $B_{T}$ is a block for $F, B_{r}$ must be a block for $f_{r}$ at $x_{r}$. However $B_{r}$ must also be a min block, otherwise, by replacing it by a strictly smaller block $B_{r}^{\prime}$, the block $B_{r}^{\prime}\left(B_{v}: v \in C(r)\right)$ will be strictly smaller than $B_{T}$.

We have shown that each min-block $B_{T}$ may be decomposed as a composition of min-blocks. By a similar argument, if $B_{T}$ is a composition of min-blocks, then it is a min-block for $F$. This shows that $\partial \mathcal{B}$ is well-behaved.

The assemblages $\mathcal{W}, \mathcal{W}^{*}$ are well-behaved:

Let $T$ be an indexed tree and let $\left(f_{v}: v \in \operatorname{Int}(T)\right)$ be a boolean function ensemble with composition $F$. Let $\mathcal{B}_{T}$ be the set of min blocks for $F$ at input $x$. For each $v \in \operatorname{Int}(T)$, let $\mathcal{B}_{v}$ be the set of min blocks for $f_{v}$ at input $x_{v}$. We just proved that $\mathcal{B}_{T}=\odot_{T}\left(\mathcal{B}_{v}: v \in \operatorname{Int}(T)\right)$. By the second part of Proposition A, for each $h_{T}$ which is a fractional hitting set for the hygergraph $\mathcal{B}_{T}$, there exists a composed fractional hitting set $h_{T}^{\prime}=\odot_{T}\left(h_{v}: v \in \operatorname{Int}(T)\right)$ such that $h_{T}^{\prime} \leq h_{T}$ (pointwise) and each $h_{v}$ is a fractional hitting set for $\mathcal{B}_{v}$. Thus, each minimal hitting set may be decomposed as a composition of hitting sets. This proves property (2). For property (1), assume that $h_{v}$ is a fractional hitting set for $\mathcal{B}_{v}$ for each $v \in \operatorname{Int}(T)$. It follows by Proposition A that $\odot_{T}\left(h_{v}: v \in \operatorname{Int}(T)\right)$ is a fractional hitting set for $\mathcal{B}_{T}$. This shows that $\mathcal{W}^{*}$ is well-behaved. The same argument works for boolean valued hitting sets, thus $\mathcal{W}$ is well-behaved.

The assemblage $\Psi$ is well-behaved: If $x$ is an input to $F$, then $\Psi_{x}(F)$ consists of a single set (the set of sensitive indices) and this set will precisely be the composition of the sets $\Psi_{x_{v}}\left(f_{v}\right)$.

Lemma 7. Let $f_{1}, \ldots, f_{k}$ be a sequence of boolean functions and let $F$ be their composition. 
- If $\vec{\alpha}_{1}, \ldots, \vec{\alpha}_{k}$ are assignment selectors, where $\vec{\alpha}_{i}$ is $f_{i}$-compatible, then $\vec{\alpha}:=\vec{\alpha}_{1} \circ \cdots \circ \vec{\alpha}_{k}$ is an F-compatible assignment selector.

- There are assignment selectors $\vec{\alpha}_{1}, \ldots, \vec{\alpha}_{k}$, where $\vec{\alpha}_{i}$ is $f_{i}$-compatible, such that $\vec{\alpha}:=\overrightarrow{\alpha_{1}} \circ \cdots \circ \overrightarrow{\alpha_{k}}$ is an m-optimal selector for $F$.

Proof. The first part follows from the second part of Proposition 4. For the second part of the present lemma, we first prove the case of $k=2$, the full statement will follow by induction. Let $F=f_{1} \circ f_{2}$ where each $f_{i}$ is a function on rooted star $T_{i}$, and let $T=T_{1} \circ T_{2}$. Let $\vec{\alpha}=\left(\alpha^{0}, \alpha^{1}\right)$ be an $m$-optimal selector for the function $F$. Let $\left(b^{c}(v): v \in \operatorname{Int}(T)\right)$ be the boolean $T$-labeling induced by evaluating $F\left(\alpha^{c}\right)$ and let $\left(b_{v}^{c}: v \in \operatorname{Int}(T)\right)$ be the corresponding assignment ensemble.

We choose $\vec{\beta}_{2}$ be any $m$-optimal selector for the function $f_{2}$, and set $\vec{\beta}_{1}:=\left(b_{r}^{0}, b_{r}^{1}\right)$. Our goal is to prove that $\vec{\beta}=\vec{\beta}_{1} \circ \vec{\beta}_{2}$ is also an $m$-optimal selector for $F$.

Writing $\vec{\beta}$ as $\left(\beta^{0}, \beta^{1}\right)$, we prove that $m_{\beta^{0}}(F) \geq m_{\alpha^{0}}(F)$; the analogous result for $\beta^{1}$ follows similarly. The construction of $\beta^{0}$ also induces an assignment ensemble which we denote as $\left(\beta_{v}^{0}\right.$ : $v \in \operatorname{Int}(T))$. Fix a minimum size weight function $w \in \mathcal{A}_{\beta^{0}}(F)$. We show how to modify $w$ to obtain a weight function $w^{\prime} \in \mathcal{A}_{\alpha^{0}}(F)$ of size at most $|w|$. This will prove the lemma, since then $m_{\alpha^{0}}(F) \leq\left|w^{\prime}\right| \leq|w|=m_{\beta^{0}}(F)$.

Since $\mathcal{A}$ is well-behaved and $w$ is minimal, we know that $w=w_{r}\left(w_{v}: v \in C(r)\right)$ for some choices of $w_{r} \in \mathcal{A}_{\beta_{r}^{0}}\left(f_{1}\right)$ and $w_{v} \in \mathcal{A}_{\beta_{v}^{0}}\left(f_{2}\right)$. Note that for each $v, f_{2}\left(\alpha_{v}^{0}\right)=f_{2}\left(\beta_{v}^{0}\right)$. It follows that $m_{\alpha_{v}^{0}}\left(f_{2}\right) \leq m_{\beta_{v}^{0}}\left(f_{2}\right)$ (because $\vec{\beta}_{2}$ is an $m$-optimal selector for $\left.f_{2}\right)$. Hence, we can find $\rho_{v} \in \mathcal{A}_{\alpha_{v}^{0}}\left(f_{2}\right)$ such that $\left|\rho_{v}\right| \leq\left|w_{v}\right|$. Having found the functions $\rho_{v}$, we set $w^{\prime}:=w_{r}\left(\rho_{v}: v \in C(r)\right)$ which will be an element in the assemblage $\mathcal{A}_{\alpha_{0}}(F)$. Moreover,

$$
\left|w^{\prime}\right|=\sum_{v \in C(r)} w_{r}(v)\left|\rho_{v}\right| \leq \sum_{v \in C(r)} w_{r}(v)\left|w_{v}\right|=|w| .
$$

To complete the proof for general $k$ we view a composed function $F=f_{1} \circ \cdots \circ f_{k}$ as $f_{1} \circ F_{k-1}$, where $F_{k-1}=f_{2} \circ \cdots \circ f_{k}$. By induction on $k$, there is an $m$-optimal selector for $F_{k-1}$ of the form $\vec{\alpha}=\vec{\alpha}_{2} \circ \cdots \circ \vec{\alpha}_{k}$. We may then repeat the proof of the case of height 2 , where we view $f_{2}$ as the function $F_{k-1}$ and choose $\vec{\beta}_{2}:=\vec{\alpha}$. Then there is an $m$-optimal selector for $F$ which is of the form $\overrightarrow{\beta_{1}} \circ \overrightarrow{\beta_{2}}=\overrightarrow{\beta_{1}} \circ \vec{\alpha}_{2} \circ \cdots \vec{\alpha}_{k}$.

We now turn to the proof of Lemma 8. Recall that an AW-selector pair over index set $I$ is a pair $(\vec{\alpha}, \vec{w})$ consisting of an assignment selector $\vec{\alpha}$ over $I$ and a weight function selector $\vec{w}$ over $I$. We need to define the uniform composition of $A W$-selector pairs. Let $\overrightarrow{\alpha_{1}}, \cdots, \overrightarrow{\alpha_{k}}$ be assignment selectors and $\overrightarrow{w_{1}}, \cdots, \overrightarrow{w_{k}}$ be weight function selectors over $L\left(T_{1}\right), \cdots, L\left(T_{k}\right)$ respectively. We define $\left(\overrightarrow{\alpha_{1}}, \overrightarrow{w_{1}}\right) \circ \cdots \circ\left(\overrightarrow{\alpha_{k}}, \overrightarrow{w_{k}}\right)$ to be the pair $(\vec{\alpha}, \vec{w})$ where $\vec{\alpha}$ is the assignment selector $\overrightarrow{\alpha_{1}} \circ \cdots \circ \overrightarrow{\alpha_{k}}$, and $\vec{w}=\left(w^{0}, w^{1}\right)$ is a weight function selector defined in the following manner. Each of $w^{0}$ and $w^{1}$ are defined, respectively, as compositions of weight function $T$-ensembles $w_{T}^{0}$ and $w_{T}^{1}$. To construct these ensembles, first recall from Section 2.8 that each component $\alpha^{c}$ (for $c \in\{0,1\}$ ) of the composition $\vec{\alpha}$ is naturally associated to a boolean $T$-labeling $b_{T}^{c}=\left(b^{c}(v): v \in \operatorname{Int}(T)\right)$. We use the labeling $b_{T}^{c}$ to define the ensemble $w_{T}^{c}=\left(w_{v}^{c}: v \in \operatorname{Int}(T)\right)$ where for node $v$ is at level $m$ (treating the root as level 1), the function $w_{v}^{c}$ is a copy of either $w_{m}^{0}$ or $w_{m}^{1}$ depending on whether $b^{c}(v)=0$ or 1. 
Lemma 8. Let $f_{1}, \ldots, f_{k}$ be a sequence of boolean functions and let $F$ be their composition. Let $\vec{\alpha}_{1}, \ldots, \vec{\alpha}_{k}$ be assignment selectors such that each $\vec{\alpha}_{i}$ is $f_{i}$-compatible. Then the following hold:

- For any sequence $\vec{w}_{1}, \ldots, \vec{w}_{k}$ of weight function selectors, where $\left(\vec{\alpha}_{i}, \vec{w}_{i}\right)$ is $\left(f_{i}, \mathcal{A}\right)$ compatible, the composition $(\vec{\alpha}, \vec{w}):=\left(\vec{\alpha}_{1}, \vec{w}_{1}\right) \circ \cdots \circ\left(\vec{\alpha}_{k}, \vec{w}_{k}\right)$ is $(F, \mathcal{A})$-compatible.

- There are weight function selectors $\vec{w}_{1}, \cdots, \vec{w}_{k}$ such that the weight function $\vec{w}$ that comes from the composition $(\vec{\alpha}, \vec{w}):=\left(\overrightarrow{\alpha_{1}}, \vec{w}_{1}\right) \circ \cdots \circ\left(\vec{\alpha}_{k}, \vec{w}_{k}\right)$ is an m-optimal weight function selector for $F$ at $\vec{\alpha}$.

Proof. In the proof of both statements let $T=T_{1} \circ \cdots \circ T_{k}$ be the indexed tree for the function $F$. Also let $(b(v): v \in T)$ be the boolean $T$-labeling induced by $\alpha^{0}$, and let $\left(\alpha_{v}^{0}: v \in \operatorname{Int}(T)\right)$ be the corresponding assignment ensemble.

For the first part, it follows from Lemma 7 that $\vec{\alpha}$ is $F$-compatible. Recall the construction of $\vec{w}=\left(w^{0}, w^{1}\right)$; in particular the weight function $w^{0}$ is the composition of the weight function ensemble $\left(w_{v}: v \in \operatorname{Int}(T)\right)$ where, if $v$ is at depth $i\left(i=1\right.$ being the root), then $w_{v}$ is a copy of $w_{i}^{b(v)}$. Also, the assignment $\alpha_{v}^{0}$ is the assignment $\alpha_{i}^{b(v)}$. Since $\left(\vec{\alpha}_{i}, \vec{w}_{i}\right)$ is a compatible pair for each $i$, it follows that $w_{v} \in \mathcal{A}_{\alpha_{v}^{0}}\left(f_{i}\right)$ for each $v$. Because $\mathcal{A}$ is well-behaved, we have $w^{0}=\odot_{T}\left(w_{v}: v \in\right.$ $\operatorname{Int}(T)) \in \mathcal{A}_{\alpha^{0}}(F)$. The exact same proof shows that $w^{1} \in \mathcal{A}_{\alpha^{1}}\left(f^{(k)}\right)$. This proves that $(\vec{\alpha}, \vec{w})$ is an $F$-compatible pair.

Now we prove the second statement. Again we prove the case $k=2$, letting the general case follow by induction. Let $\vec{\alpha}=\left(\alpha^{0}, \alpha^{1}\right)$. To construct $\vec{w}$, we will choose $\vec{w}_{2}$ to be any $m$-optimal weight function selector for $f_{2}$ at $\vec{\alpha}_{2}$ and construct $\vec{w}_{1}=\left(w_{1}^{0}, w_{1}^{1}\right)$.

We first construct $w_{1}^{0}$. Let $w^{*}$ be any minimum sized weight function in $\mathcal{A}_{\alpha^{0}}(f)$. Because $\mathcal{A}$ is well-behaved and $w^{*}$ is minimal, we may decompose $w^{*}=w_{r}^{*}\left(w_{v}^{*}: v \in C(r)\right)$ where $w_{r}^{*} \in \mathcal{A}_{\alpha_{r}^{0}}\left(f_{1}\right)$ and $w_{v}^{*} \in \mathcal{A}_{\alpha_{v}^{0}}\left(f_{2}\right)$. We will set $w_{1}^{0}:=w_{r}^{*}$ and check that it satisfies the properties we need. Consider $w^{\prime}:=w_{r}^{*}\left(w_{v}: v \in C(r)\right)$ where $w_{v}:=w_{2}^{b(v)}$. Note that $w^{\prime} \in \mathcal{A}_{\alpha^{0}}(f)$ because $\mathcal{A}$ is well-behaved and moreover it has size

$$
\left|w^{\prime}\right|=\sum_{v \in C(r)} w_{r}^{*}(v)\left|w_{2}^{b(v)}\right| \leq \sum_{v \in C(r)} w_{r}^{*}(v)\left|w_{v}^{*}\right|=\left|w^{*}\right|
$$

Here the inequality follows from the fact that $w_{2}^{0}$ and $w_{2}^{1}$ have minimum sizes in the families $\mathcal{A}_{\alpha_{2}^{0}}\left(f_{2}\right)$ and $\mathcal{A}_{\alpha_{2}^{1}}\left(f_{2}\right)$ respectively.

In the same manner construct $w_{1}^{1}$. Finally, set $(\vec{\alpha}, \vec{w}):=\left(\vec{\alpha}_{1}, \vec{w}_{1}\right) \circ\left(\vec{\alpha}_{2}, \vec{w}_{2}\right)$ where $\vec{w}=\left(w^{0}, w^{1}\right)$. Then by construction, $w^{0}=w^{\prime}$, and we have shown $\left|w^{0}\right| \leq\left|w^{*}\right|$. Thus, $w^{0}$ must have minimum size in the family $\mathcal{A}_{\alpha^{0}}(f)$. By the same argument, the function $w^{1}$ will have minimum size in the family $\mathcal{A}_{\alpha^{1}}(f)$. Therefore, $\vec{w}$ is an $m$-optimal selector for $f$ at $\vec{\alpha}$ as desired.

To see the induction step, view $F=f_{1} \circ \cdots \circ f_{k}$ as a composition of two functions $f_{1} \circ F_{k-1}$ where $F_{k-1}=f_{2} \circ \cdots \circ f_{k}$. We now use the same construction, only noting that by induction on $\mathrm{k}$ we may choose $\vec{w}_{2}$ to a composition of AW selector-pairs.

We now show that multiplication of profile matrices encapsulates crucial information about AW selector-pair composition. The following definitions will be helpful.

- Profile vector of a weight function $w$ on assignment $x$. The profile of $(x, w)$ is the pair $p_{x}(w):=\left(p_{0}, p_{1}\right)$ where $p_{0}:=\sum_{i: x_{i}=0} w_{i}$ and $p_{1}:=\sum_{i: x_{i}=1} w_{i}$. 
- Profile vector family $P_{x}(f)$ for the assignment $x$ and assemblage $\mathcal{A}$. This is the set of all profile vectors $p_{x}(w)$ where $w$ ranges over weight functions in $\mathcal{A}_{x}(f)$.

For any profile matrix $M:=M_{\vec{\alpha}, \vec{w}}$, the first row of $M$ is the profile vector $p_{\alpha^{0}}\left(w^{0}\right)$ and the second row is the profile vector $p_{\alpha^{1}}\left(w^{1}\right)$.

Proposition 10. For any sequence $\left(\vec{\alpha}_{1}, \vec{w}_{1}\right), \ldots,\left(\vec{\alpha}_{k}, \vec{w}_{k}\right)$ of $A W$ selector-pairs, if $(\vec{\alpha}, \vec{w})$ is their composition then the profile matrix $M_{\vec{\alpha}, \vec{w}}$ is given by:

$$
M_{\vec{\alpha}, \vec{w}}=M_{\vec{\alpha}_{1}, \vec{w}_{1}} \cdots M_{\vec{\alpha}_{k}, \vec{w}_{k}} .
$$

Proof. We prove the special case where $k=2$, the general case will then follow by induction. Let $(\vec{\alpha}, \vec{w})=\left(\vec{\alpha}_{1}, \vec{w}_{1}\right) \circ\left(\vec{\alpha}_{2}, \vec{w}_{2}\right)$ be the composed pair, where $\vec{w}=\left(w^{0}, w^{1}\right)$ and $\vec{\alpha}=\left(\alpha^{0}, \alpha^{1}\right)$, and let $T$ be the corresponding indexed tree. Let

$$
M_{\vec{\alpha}_{1}, \vec{w}_{1}}=\left[\begin{array}{cc}
a_{00} & a_{01} \\
a_{10} & a_{11}
\end{array}\right] \quad M_{\vec{\alpha}_{2}, \vec{w}_{2}}=\left[\begin{array}{ll}
b_{00} & b_{01} \\
b_{10} & b_{11}
\end{array}\right] \quad M_{\vec{\alpha}, \vec{w}}=\left[\begin{array}{cc}
c_{00} & c_{01} \\
c_{10} & c_{11}
\end{array}\right] .
$$

We check that $c_{00}=a_{00} b_{00}+a_{01} b_{10}$, the other entries will follow by similar arguments. We check this by computing the profile vector for the input $\alpha^{0}$. Let $(b(v): v \in T)$ be the boolean $T$-labeling induced by the construction of $\alpha^{0}$ and let $\left(b_{v}: v \in \operatorname{Int}(T)\right)$ be the corresponding assignment ensemble. By construction, $b_{r}=\alpha_{1}^{0}$ and $b_{v}=\alpha_{2}^{b(v)}$ for $v \in C(r)$. Recall that $w^{0}:=w_{r}\left(w_{v}: v \in\right.$ $C(r))$, where $w_{r}=w_{1}^{0}$ and $w_{v}:=w_{2}^{b(v)}$ for $v \in \operatorname{Int}(T)$.

The profile vector $p_{\alpha^{0}}\left(w^{0}\right):=\left[c_{00}, c_{01}\right]$. In particular, $c_{00}=\sum_{l \in L(T): \alpha^{0}(l)=0} w^{0}(l)$. Thus, we have

$$
\begin{aligned}
& c_{00}=\sum_{\substack{l \in L(T) \\
\alpha^{0}(l)=0}} w^{0}(l) \\
& =\sum_{v \in C(r)} \sum_{\substack{l \in C(v) \\
\alpha^{0}(l)=0}} w_{r}(v) w_{v}(l) \\
& =\sum_{\substack{v \in C(r) \\
b_{r}(v)=0}} w_{r}(v) \sum_{\substack{l \in C(v) \\
b_{v}(l)=0}} w_{2}^{0}(l)+\sum_{\substack{v \in C(r) \\
b_{r}(v)=1}} w_{r}(v) \sum_{\substack{l \in C(v) \\
b_{v}(l)=0}} w_{2}^{1}(l) \\
& =\sum_{\substack{v \in C(r) \\
\alpha_{1}^{0}(v)=0}} w_{r}(v) \sum_{\substack{l \in C(v) \\
\alpha_{2}^{0}(l)=0}} w_{2}^{0}(l)+\sum_{\substack{v \in C(r) \\
\alpha_{1}^{0}(v)=1}} w_{r}(v) \sum_{\substack{l \in C(v) \\
\alpha_{2}^{1}(l)=0}} w_{2}^{1}(l) \\
& =\sum_{\substack{v \in C(r) \\
\alpha_{1}^{0}(v)=0}} w_{1}^{0}(v) b_{00}+\sum_{\substack{v \in C(r) \\
\alpha_{1}^{0}(v)=1}} w_{1}^{0}(v) b_{10} \\
& =a_{00} b_{00}+a_{01} b_{10} \text {. }
\end{aligned}
$$

We now present the two main lemmas which imply our main result. The proofs reduce the statements to two claims regarding the largest eigenvalue of the product of certain matrices which we delay for the next section. 
Lemma 13. For any boolean function $f$ and $k \in \mathbb{N}$

$$
m\left(f^{(k)}\right) \geq \hat{m}(f)^{k} / 2 .
$$

Proof. Let $\lambda:=\hat{m}(f)$. Let $\vec{\beta}$ be an assignment selector for which $\hat{m}_{\vec{\beta}}(f)=\lambda$. Let $\vec{\alpha}:=\vec{\beta}^{(k)}$. By Corollary 11 and Fact 12, the claim will follow from showing that, for any sequence of profile matrices $M_{i} \in \mathcal{M}_{\vec{\beta}}\left(f^{(k)}\right)$, we have

$$
\rho\left(M_{1} M_{2} \cdots M_{k}\right) \geq \lambda^{k} .
$$

Let $\left\{M_{i}\right\}_{i=1}^{k}$ be any such sequence of matrices and let $M$ be their product. Because of our choice of $\vec{\beta}$, we know that $\rho\left(M_{i}\right) \geq \lambda$ for each $i$. In general, this is not enough to guarantee that $\rho(M) \geq \lambda^{k}$. However, these matrices contain additional structure which will allow us to make such a conclusion.

Recall that each profile matrix $M_{i} \in \mathcal{M}_{\vec{\beta}}(f)$ corresponds to a weight function selector $\vec{w}_{i}$ which is $\vec{\beta}$-compatible. For each $i, j \in[k]$ let $M_{i j}$ denote the matrix who's first row is the first row of $M_{i}$ (i.e., the profile vector $p_{\beta^{0}}\left(w_{i}^{0}\right)$ ), and who's second row is the second row of $M_{j}$ (i.e., the profile vector $\left.p_{\beta^{1}}\left(w_{j}^{1}\right)\right)$. Then $M_{i j}$ is precisely the profile matrix $M_{\vec{\beta}, \vec{w}_{i j}}$ where $\vec{w}_{i j}=\left(w_{i}^{0}, w_{j}^{1}\right)$. In particular, each $M_{i j} \in \mathcal{M}_{\vec{\beta}}(f)$ and $\rho\left(M_{i j}\right) \geq \lambda$ by the definition of $\lambda$. Noting this property, we apply Lemma 17 (see the following section 3.3 ) and conclude that

$$
\rho(M) \geq \lambda^{k} .
$$

Lemma 14. For any boolean function $f$ on $n$ variables and $k \in \mathbb{N}$,

$$
m\left(f^{(k)}\right) \leq 2 n k(\hat{m}(f))^{k-1} .
$$

Proof. Let $\lambda:=\hat{m}(f)$. Take $\vec{\alpha}$ which is an $m$-optimal selector for $f^{(k)}$. By lemma 7, we may assume that $\vec{\alpha}=\overrightarrow{\alpha_{1}} \circ \overrightarrow{\alpha_{2}} \circ \cdots \circ \overrightarrow{\alpha_{k}}$. By Corollary 11, the claim will follow by exhibiting matrices $M_{i} \in \mathcal{M}_{\alpha_{i}}(f)$ such that

$$
\left\|M_{1} M_{2} \cdots M_{k}\right\|_{\infty} \leq n k \lambda^{k-1} .
$$

Let $\mathcal{U}_{i}$ be the profile vector family $P_{\alpha_{i}^{0}}(f)$, and let $\mathcal{V}_{i}=P_{\alpha_{i}^{1}}(f)$. When considering the possible choices of $M_{i} \in \mathcal{M}_{\vec{\alpha}_{i}}(f)$, the set $\mathcal{U}_{i}$ is the set of possible first rows of $M_{i}$. Likewise, $\mathcal{V}_{i}$ is the set of possible second rows of $M_{i}$. One may hope to use the definition of $\lambda$ and choose each $M_{i}$ such that $\rho\left(M_{i}\right) \leq \lambda$. This in general though is not enough to bound all entries in the product $M_{1} \cdots M_{k}$. Once again we need to use additional structure of these matrix families. Note crucially that, for each $i, j \in[k]$, there exists $u \in \mathcal{U}_{i}$ and $v \in \mathcal{V}_{j}$ such that

$$
\rho\left(\left[\begin{array}{l}
u \\
v
\end{array}\right]\right) \leq \lambda .
$$

This follows by the definition of $\lambda$ and the fact that the set of profile matrices

$$
\mathcal{M}_{\vec{\alpha}_{i j}}(f)=\left\{\left[\begin{array}{l}
u \\
v
\end{array}\right] \mid u \in \mathcal{U}_{i}, v \in \mathcal{V}_{j}\right\}
$$

where $\vec{\alpha}_{i j}:=\left(\alpha_{i}^{0}, \alpha_{j}^{1}\right)$. By Corollary 21 (see section [3.3), there exists matrices $M_{1}, M_{2}, \cdots, M_{k}$, where $M_{i} \in \mathcal{M}_{\vec{\alpha}_{i}}(f)$ for each $i$, such that $\left\|M_{1} M_{2} \cdots M_{k}\right\|_{\infty} \leq n k \lambda^{k-1}$. 


\subsection{Facts about non-negative matrices}

In this subsection, we prove Lemmas 17 and 18 which were used in the previous subsection. We will need the following well-known facts about $2 \times 2$ non-negative matrices that follow from PerronFrobenius theory (for omitted proofs see, e.g., Mey00, Chapter 8]).

Fact 16. Fix $A \in \mathbb{R}_{\geq 0}^{2 \times 2}$. We have the following:

1. There exists a non-zero $z \geq 0$ s.t. $A z=\rho(A) z$.

2. For $\lambda \in \mathbb{R}$, the following are equivalent: (1) $\rho(A) \geq \lambda$, (2) $\exists x \geq 0$ such that $x \neq 0$ and $A x \geq \lambda x$, and (3) For every $\varepsilon>0$, there exists an $x>0$ such that $A x \geq(\lambda-\varepsilon) x$.

3. For $\lambda \in \mathbb{R}$, we have $\rho(A) \leq \lambda$ iff for every $\varepsilon>0$, there is an $x>0$ s.t. $A x \leq(\lambda+\varepsilon) x$.

4. $\|A\|_{\infty} \geq \rho(A) / 2$.

5. $\lim _{k \rightarrow \infty}\left\|A^{k}\right\|_{\infty}^{1 / k}=\rho(A)$.

We can now prove the two main lemmas of this subsection.

Lemma 17. Let $M_{1}, \ldots, M_{k} \in \mathbb{R}_{\geq 0}^{2 \times 2}$ and let $M:=M_{1} \cdots M_{k}$. For each $i, j \in[k]$, let $M_{i, j}$ denote the matrix whose first and second rows are the first row of $M_{i}$ and the second row of $M_{j}$ respectively. If $\rho\left(M_{i, j}\right) \geq \lambda \geq 0$ for each $i, j \in[k]$, then $\rho(M) \geq \lambda^{k}$.

Proof. The lemma is trivial for $\lambda=0$. Thus, we assume that $\lambda>0$. By dividing each matrix through by $\lambda$, we can assume w.l.o.g. that $\lambda=1$. In this case, we need to show that $\rho(M) \geq 1$.

By Fact 16, we can show that $\rho(M) \geq 1$ by showing that there exists a non-zero $z \in \mathbb{R}^{2}$ s.t. $z \geq 0$ and $M z \geq z$. To do this, it suffices to produce a $z$ as above s.t. $M_{i} z \geq z$ for each $i$.

Denote by $u_{i}=\left(u_{i, 1}, u_{i, 2}\right)$ and $v_{i}=\left(v_{i, 1}, v_{i, 2}\right)$ the first and second rows (respectively) of $M_{i}$. We need $M_{i} z \geq z$, which is the same as requiring that $\left\langle u_{i}^{\prime}, z\right\rangle \geq 0$ and $\left\langle v_{i}^{\prime}, z\right\rangle \geq 0$ for every $i$, where $u_{i}^{\prime}=\left(u_{i, 1}-1, u_{i, 2}\right)^{T}$ and $v_{i}^{\prime}=\left(v_{i, 1}, v_{i, 2}-1\right)^{T}$. Clearly, if $u_{i}^{\prime}$ or $v_{i}^{\prime}$ is non-negative, the corresponding constraint is trivial (since we are looking for $z \geq 0$ ). Let $P$ and $Q$ denote the set of $i$ where $u_{i, 1}<1$ and $v_{i, 2}<1$ respectively.

Thus the constraint corresponding to $u_{i}^{\prime}$ for $i \in P$ may be rewritten as $z_{1} \leq\left(u_{i, 2} /\left(1-u_{i, 1}\right)\right) \cdot z_{2}$. Clearly, this constraint gets strictly harder to satisfy as the parameter $u_{i, 2} /\left(1-u_{i, 1}\right)$ gets smaller and therefore, to satisfy all the constraints indexed by $P$, it suffices to satisfy just the constraint corresponding to $i_{0} \in P$ for which this parameter is minimized. Similarly, there is a $j_{0} \in Q$ s.t. any non-negative $z$ that satisfies $\left\langle v_{j_{0}}^{\prime}, z\right\rangle \geq 0$ automatically satisfies all the other constraints indexed by $Q$. However, we know that $\rho\left(M_{i_{0}, j_{0}}\right) \geq 1$ and hence by Fact 16, there is some non-zero $z \in \mathbb{R}_{\geq 0}^{2}$ s.t. $M_{i_{0}, j_{0}} z \geq z$ and thus $\left\langle u_{i_{0}}^{\prime}, z\right\rangle \geq 0$ and $\left\langle v_{j_{0}}^{\prime}, z\right\rangle \geq 0$. This $z$ satisfies all the constraints and hence has the property that $M_{i} z \geq z$ for each $i \in[k]$.

Given $u, v \in \mathbb{R}^{2}$, we denote by $\left[\begin{array}{l}u \\ v\end{array}\right]$ the $2 \times 2$ matrix whose first and second rows are $u$ and $v$ respectively.

Lemma 18. Assume we have compact subsets $U_{1}, \ldots, U_{k}, V_{1}, \ldots, V_{k} \subseteq \mathbb{R}_{>0}^{2}$ s.t. for each $i, j \in[k]$, there exists $u_{i, j} \in U_{i}$ and $v_{i, j} \in V_{j}$ s.t. the matrix $\left[\begin{array}{l}u_{i, j} \\ v_{i, j}\end{array}\right]$ satisfies $\rho\left(\left[\begin{array}{l}u_{i, j} \\ v_{i, j}\end{array}\right]\right) \leq \lambda$. Then, there exist $u_{i} \in U_{i}$ and $v_{i} \in V_{i}$ for each $i \in[k]$ s.t. the matrices $M_{i}:=\left[\begin{array}{l}u_{i} \\ v_{i}\end{array}\right]$ and $M^{[i, j]}:=M_{i} \cdot M_{i+1} \cdots M_{j}$ for $i \leq j \in[k]$ satisfy $\rho\left(M^{[i, j]}\right) \leq \lambda^{j-i+1}$. 
Proof. We will show that for each $\varepsilon>0$, there is a choice of $u_{i} \in U_{i}, v_{i} \in V_{i}(i \in[k])$ so that for $M_{i}:=\left[\begin{array}{l}u_{i} \\ v_{i}\end{array}\right]$ and $M^{[i, j]}:=M_{i} \cdots M_{j}$, we have

$$
\rho\left(M^{[i, j]}\right)=\rho\left(M_{i} \cdots M_{j}\right) \leq(\lambda+\varepsilon)^{j-i+1} .
$$

for each $i, j \in[k]$ with $i<j$. Since the sets $U_{i}, V_{i}$ for $i \in[k]$ are all compact and $\rho: \mathbb{R}^{2 \times 2} \rightarrow \mathbb{R}$ is a continuous function, a standard argument shows there must be a choice of these vectors so that $M$ as defined above in fact satisfies the requirements of the lemma.

Fix $\varepsilon>0$ and let $\lambda^{\prime}=\lambda+\varepsilon$. We first show how to choose $u_{i}, v_{i}(i \in[k])$ and $z \in \mathbb{R}_{>0}^{2}$ such that for each $i, M_{i}:=\left[\begin{array}{l}u_{i} \\ v_{i}\end{array}\right]$ satisfies $M_{i} z \leq \lambda^{\prime} z$. We then show how this implies (3)).

Claim 19. There exist $u_{i} \in U_{i}$ and $v_{i} \in V_{i}$ for each $i \in[k]$ and $a z \in \mathbb{R}_{>0}^{2}$ such that for each $i \in[k]$, we have $M_{i} z \leq \lambda^{\prime} z$, where $M_{i}$ is as defined above.

Proof of Claim [19. The vectors $u_{1}, \ldots, u_{k}, v_{1}, \ldots, v_{k}$ and $z \in \mathbb{R}_{>0}^{2}$ that we choose will in fact have the stronger property that for each $i, j \in[k]$, we will have $M_{i, j} z \leq z$, where $M_{i, j}:=\left[\begin{array}{l}u_{i} \\ v_{j}\end{array}\right]$. Let us fix $i, j \in[k]$ and consider the problem of coming up with such a $u_{i}, v_{j}$, and $z$. Therefore, we want $u_{i} \in U_{i}$ and $v_{j} \in V_{j}$ s.t.

$$
\left\langle u_{i}, z\right\rangle \leq \lambda^{\prime} z_{1} \quad\left\langle v_{j}, z\right\rangle \leq \lambda^{\prime} z_{2}
$$

We can rewrite the above constraints on $z$ as

$$
\left\langle u_{i}^{\prime}, z\right\rangle \leq 0 \quad\left\langle v_{j}^{\prime}, z\right\rangle \leq 0
$$

where $u_{i}^{\prime}:=\left(\lambda^{\prime}-u_{i, 1}, u_{i, 2}\right)$ and $v_{j}^{\prime}:=\left(v_{j, 1}, \lambda^{\prime}-v_{j, 2}\right)$. Consider the set of constraints $\left\{\left\langle u_{i}^{\prime}, z\right\rangle \leq\right.$ $\left.0 \mid u_{i} \in U_{i}\right\}$. Note that this set of constraints has the property is that there is a weakest constraint: more precisely, there exists a $u_{i} \in U_{i}$ s.t. for any $z>0$, if there exists a $\bar{u}_{i} \in U_{i}$ s.t. $\left\langle\bar{u}_{i}^{\prime}, z\right\rangle \leq 0$, then $\left\langle u_{i}^{\prime}, z\right\rangle \leq 0$ as well. Similarly, we also have a $v_{j} \in V_{j}$.

We need a crucial observation regarding the vectors $u_{i}, v_{i}$ chosen above. By the assumptions of Lemma 18, for every $i, j \in[k]$, we know that for each $i, j \in[k]$, there is some choice of $u_{i, j} \in U_{i}$ and $v_{j, i} \in V_{j}$ so that $\rho\left(\left[\begin{array}{l}u_{i, j} \\ v_{i, j}\end{array}\right]\right) \leq \lambda$. By Fact [16, this means that there is some $z_{i, j} \in \mathbb{R}_{>0}^{2}$ s.t. $\left[\begin{array}{l}u_{i, j} \\ v_{i, j}\end{array}\right] z_{i, j} \leq \lambda^{\prime} z_{i, j}$, which is equivalent to saying that $\left\langle u_{i, j}^{\prime}, z_{i, j}\right\rangle \leq 0$ and $\left\langle v_{i, j}^{\prime}, z_{i, j}\right\rangle \leq 0$. But this implies that $\left\langle u_{i}, z_{i, j}\right\rangle \leq 0$ and $\left\langle v_{j}, z_{i, j}\right\rangle \leq 0$ as well. Thus, we have shown that

Observation 20. For every $i, j \in[k]$, there exists a $z_{i, j} \in \mathbb{R}_{>0}^{2}$ s.t. $\left\langle u_{i}^{\prime}, z_{i, j}\right\rangle \leq 0$ and $\left\langle v_{j}^{\prime}, z_{i, j}\right\rangle \leq 0$.

Now that we have chosen $u_{i}, v_{i}$ for each $i \in[k]$, we only need to choose $z \in \mathbb{R}_{>0}^{2}$ as mentioned above. Again, we need to choose $z \in \mathbb{R}_{>0}^{2}$ so that for each $i, j,\left\langle u_{i}^{\prime}, z\right\rangle \leq 0$ and $\left\langle v_{j}^{\prime}, z\right\rangle \leq 0$. Consider the sets of constraints $\left\{\left\langle u_{i}^{\prime}, z\right\rangle \leq 0 \mid i \in[k]\right\}$ and $\left\{\left\langle v_{j}^{\prime}, z\right\rangle \leq 0 \mid j \in[k]\right\}$. This time we consider the strongest constraints in these sets: in other words, we fix an $i_{0} \in[k]$ so that for any $z>0$, if $\left\langle u_{i_{0}}^{\prime}, z\right\rangle \leq 0$, then in fact $\left\langle u_{i}^{\prime}, z\right\rangle \leq 0$ for every $i \in[k]$ and a $j_{0} \in[k]$ similarly for the $v_{j}$. By Observation 20, we know that there is a $z:=z_{i_{0}, j_{0}}>0$ that satisfies these constraints and since these are the strongest constraints, we see that $z$ satisfies $M_{i, j} z \leq z$ for every $i, j \in[k]$.

Fix any $i, j \in[k]$ s.t. $i<j$ and consider $M^{[i, j]}=M_{i} \cdots M_{j}$, where the $M_{\ell}(\ell \in[k])$ are as given by Claim [19, We show $\rho\left(M^{[i, j]}\right) \leq\left(\lambda^{\prime}\right)^{j-i+1}$. By Fact [16, it suffices to obtain $z \in \mathbb{R}_{>0}^{2}$ s.t. $M^{[i, j]} \cdot z \leq\left(\lambda^{\prime}\right)^{j-i+1} z$. Consider the $z$ guaranteed to us by Claim [19, We have $M^{[i, j]} \cdot z=$ $\left(M_{i} \cdots M_{j}\right) z \leq\left(M_{i} \cdots M_{j-1}\right)\left(\lambda^{\prime} z\right) \ldots \leq\left(\lambda^{\prime}\right)^{j-i+1} z$, where the inequalities follows from the choice of $z$ and the fact that the matrices $M_{\ell}$ are all non-negative. This finishes the proof of Lemma 18. 
Corollary 21. Let $U_{1}, \ldots, U_{k}, V_{1}, \ldots, V_{k}$, and $\lambda$ be as in Lemma 18. Suppose further that, for any $i$ and any $u \in U_{i}$ and $v \in V_{i}$, the entries of $u$ and $v$ are bounded above by a constant $n$. Then there exist $u_{i} \in U_{i}$ and $v_{i} \in V_{i}$ for each $i \in[k]$ such that the matrices $M_{i}:=\left[\begin{array}{l}u_{i} \\ v_{i}\end{array}\right]$ satisfy $\left\|M_{1} M_{2} \cdots M_{k}\right\|_{\infty} \leq n k \lambda^{k-1}$.

Proof. For $i \in[k]$ let $u_{i}, v_{i}$ and $M_{i}:=\left[\begin{array}{l}u_{i} \\ v_{i}\end{array}\right]$ be the matrices guaranteed by Lemma 18, Also, let $M^{[1, i]}:=M_{1} M_{2} \cdots M_{i}$. By lemma [18, for each $i$ we have that $\rho\left(M_{i}\right) \leq \lambda$ and $\rho\left(M^{[1, i]}\right) \leq \lambda^{i}$. It is easy to check that given a $2 \times 2$ matrix $M$ with non-negative entries, if $\rho(M) \leq C$ then the diagonal entries are both $\leq C$.

We prove by induction on $i$ that the matrices $M^{[1, i]}$ are entry-wise $\leq\left[\begin{array}{c}\lambda^{i} \\ n i \lambda^{i-1}\end{array} \begin{array}{c}n i \lambda^{i-1} \\ \lambda^{i}\end{array}\right]$. The base case follows because we have $M_{1} \leq\left[\begin{array}{ll}\lambda & n \\ n & \lambda\end{array}\right]$. The diagonal entries of $M^{[1, i+1]}$ are $\leq \lambda^{i+1}$, because $\rho\left(M^{[1, i+1]}\right) \leq \lambda^{i+1}$. For the off diagonal entries, note that $M^{[1, i+1]}=M^{[1, i]} M_{i+1}$. By the inductive hypothesis, $M^{[1, i]} \leq\left[\begin{array}{cc}\lambda^{i} & n i \lambda^{i-1} \\ n i \lambda^{i-1} & \lambda^{i}\end{array}\right]$. Also because $\rho\left(M_{i}\right) \leq \lambda$ we have $M_{i} \leq\left[\begin{array}{ll}\lambda & n \\ n & \lambda\end{array}\right]$. Thus the off diagonal entries of $M^{[1, i+1]}$ are bounded above by $n \lambda^{i}+n i \lambda^{i}=n(i+1) \lambda^{i}$.

This completes the proof as then, $\left\|M^{[1, k]}\right\|_{\infty} \leq n k \lambda^{k-1}$.

\section{The behaviour of Block sensitivity under iterated composition}

In this section, we characterize the behavior of the block sensitivity $b s(f)$ under iterated composition. We show that for any function $f:\{0,1\}^{I} \rightarrow\{0,1\}$, we have $b s^{\lim }(f)=\left(b s^{*}\right)^{\lim }(f)$. We use similar notation as in the previous sections such as the concepts of indexed trees $T, T$-ensembles, and $T$-compositions, only now we will denote by $I$ to be the index set for a function $f$ (which corresponds to a rooted star $T$ ).

We state the main result of this section formally below.

Theorem 22. For any boolean function $f:\{0,1\}^{I} \rightarrow\{0,1\}$, we have bs $s^{\lim }(f)=\left(b s^{*}\right)^{\lim }(f)$.

The above is easily proved when $f$ is either monotone or anti-monotone. In this case, we know that for each $k \in \mathbb{N}, f^{(k)}$ is either monotone or anti-monotone and hence $b s\left(f^{(k)}\right)=C\left(f^{(k)}\right)$ [Nis91, BdW02. As $b s^{*}\left(f^{(k)}\right)$ is sandwiched between $b s\left(f^{(k)}\right)$ and $C\left(f^{(k)}\right)$, we have $b s\left(f^{(k)}\right)=$ $b s^{*}\left(f^{(k)}\right)$ and thus we are done. So from now on, we assume that $f$ is neither monotone nor anti-monotone.

\subsection{Some simple claims}

Recall Fekete's lemma for superadditive sequences (see, e.g., [SUB11, Section A.4]).

Lemma 23 (Fekete's lemma). Let $\left\{a_{m}\right\}_{m \in \mathbb{N}}$ be a sequence of real numbers such that for any $p, q \in \mathbb{N}, a_{p+q} \geq a_{p}+a_{q}$. Then, the limit $\lim _{k \rightarrow \infty} a_{k} / k$ exists (and is possibly infinite) and moreover, we have $\lim _{k \rightarrow \infty} a_{k} / k=\sup _{k} a_{k} / k$.

We have the following easy corollary to the above lemma for sequences that are "almost superadditive".

Corollary 24. Let $\left\{a_{m}\right\}_{m \in \mathbb{N}}$ be a sequence of real numbers such that for any $p, q \in \mathbb{N}, a_{p+q} \geq$ $a_{p}+a_{q}-c$ for some fixed $c \in \mathbb{R}^{\geq 0}$. Then, the limit $\lim _{k \rightarrow \infty} a_{k} / k$ exists. 
Proof. Consider the sequence $\left\{b_{m}\right\}_{m \in \mathbb{N}}$ defined by $b_{m}=a_{m}-c$. Then, $\left\{b_{m}\right\}_{m}$ is clearly superadditive and moreover, we have $\lim _{k \rightarrow \infty}\left(a_{k}-b_{k}\right) / k=0$. Thus, by Lemma 23, we are done.

Lemma 25. Fix any boolean function $f:\{0,1\}^{I} \rightarrow\{0,1\}$ and any $x \in\{0,1\}^{I}$. For any $M, k \geq 1$, we have $b s_{x}^{k M}(f) \geq k \cdot b s_{x}^{M}(f)$.

Proof. Given any $M$-fold packing $\mathcal{B}$ of blocks of size $s$ in $\mathcal{B}_{x}(f)$, we can construct a $k M$-fold packing of blocks $\mathcal{B}^{\prime}$ of size $k s$ in $\mathcal{B}_{x}(f)$ by simply repeating $\mathcal{B} k$ times. When $\mathcal{B}$ is chosen to be the $M$-fold packing of maximum size for $f$ at $x$, this shows that $b s_{x}^{k M}(f) \geq k|\mathcal{B}|=k \cdot b s_{x}^{M}(f)$.

The following lemma will be crucial in showing that $b s\left(f^{(k)}\right)$ grows like $b s^{*}\left(f^{(k)}\right)$.

Lemma 26. Let $g_{i}:\{0,1\}^{I_{i}} \rightarrow\{0,1\}$ ( $\left.i \in[2]\right)$ be any non-constant boolean functions. Let $G$ denote the depth-2 composition $g_{1} \circ g_{2}$ defined on the index set $I_{1} \times I_{2}$. Then, for any $b \in\{0,1\}$, we have

$$
b s_{b}(G) \geq b s_{b}^{M}\left(g_{1}\right)
$$

where $M=\min \left\{b s_{0}\left(g_{2}\right), b s_{1}\left(g_{2}\right)\right\}$.

Proof. As a short remark, we may view the index set $I_{1} \times I_{2}$ as the leaves of the tree $T=T_{1} \circ T_{2}$ where $T_{i}$ is a rooted star corresponding to the index set $I_{i}$. Also, for an assignment $x$ to $I_{1}$ and $i \in I_{1}$, we will use $x(i)$ to denote the boolean value $x$ assigns to $i$.

We prove the lemma for $b=0$; an identical proof works for $b=1$. Let $\vec{\alpha}=\left(\alpha^{0}, \alpha^{1}\right)$ be a $g_{2}$-optimal selector (so $b s_{\alpha^{b}}\left(g_{2}\right)=b s_{b}\left(g_{2}\right)$ for $b \in\{0,1\}$ ). Let $x \in g_{1}^{-1}(0)$ be chosen so that $b s_{x}^{M}\left(g_{1}\right)=b s_{0}^{M}\left(g_{1}\right)$. Consider the composed assignment $X=x \circ \vec{\alpha}$ to the input of $G$. We will show that $b s_{X}(G) \geq b s_{0}^{M}\left(g_{1}\right)$, which will prove the lemma.

For $b \in\{0,1\}$, let $\mathcal{B}_{b}$ be any maximum-sized packing in the hypergraph $\mathcal{B}_{\alpha^{b}}\left(g_{2}\right)$. Note that $\min \left\{\left|\mathcal{B}_{0}\right|,\left|\mathcal{B}_{1}\right|\right\}=M$. Let $\mathcal{B}$ be a maximum-sized $M$-fold block packing in $\mathcal{B}_{x}\left(g_{1}\right)$. We now give an algorithm that constructs a block packing $\mathcal{B}^{\prime}$ in $\mathcal{B}_{X}(G)$ such that $\left|\mathcal{B}^{\prime}\right|=|\mathcal{B}|=b s_{0}^{M}\left(g_{1}\right)$.

For each $i \in I_{1}$, the algorithm maintains a packing $\mathcal{B}^{i}$ of the hypergraph $\mathcal{B}_{\alpha^{x(i)}}\left(g_{2}\right)$. We initialize $\mathcal{B}^{i}$ to be $\mathcal{B}_{x(i)}$. We now perform the following for each block $B \in \mathcal{B}$ (considered in some arbitrary order):

- For each $i \in I_{1}$, define the set $B_{i}$ to be empty if $i \notin B$, and to be a member of $\mathcal{B}_{x(i)}$ if $i \in B$. Let $B^{\prime}$ be the composition $B\left(B^{i}: i \in I_{1}\right.$ ). (Here the composition of blocks is subset composition which, as defined in Section 2.7, is obtained by viewing each block as a boolean weight function, and using composition of weight functions.)

- For $i \in B$, the set $\mathcal{B}^{i}$ is updated to $\mathcal{B}^{i} \backslash\left\{B^{i}\right\}$.

The blocks $B^{\prime}$ thus constructed are easily seen to belong to $\mathcal{B}_{X}(G)$ and to be pairwise disjoint and so form a block packing in $\mathcal{B}_{X}(G)$. Provided that we can carry out the process for each block $B \in \mathcal{B}$ we get the correct number of blocks in our packing. We need to verify that the first step inside the loop is well-defined, for which we require that when the block $B$ is considered, for each $i \in B, \mathcal{B}_{(x(i)}$ must be nonempty so that we can select $B_{i}$. This is true since $\mathcal{B}_{x(i)}$ initially has size at least $M$, and decreases by 1 each time we consider a block $C$ that contains $i$, and $i$ belongs to at most $M$ blocks of $\mathcal{B}$. 
Lemmas 25] and 26 yield the following.

Corollary 27. Let $f$ be such that $\min \left\{b s_{0}(f), b s_{1}(f)\right\} \geq 2$. Then, $\min \left\{b s_{0}\left(f^{(k)}\right), b s_{1}\left(f^{(k)}\right)\right\} \geq 2^{k}$. In particular, $\min \left\{b s_{0}\left(f^{(k)}\right), b s_{1}\left(f^{(k)}\right)\right\}$ goes to infinity as $k \rightarrow \infty$.

Proof. By Lemmas 26 and 25, for any $k \geq 1$, we have $b s\left(f^{(k+1)}\right) \geq b s^{2}\left(f^{(k)}\right) \geq 2 b s\left(f^{(k)}\right)$. Hence, by induction on $k$, we have the claim.

\subsection{Proof of Theorem 22}

Throughout $f:\{0,1\}^{I} \rightarrow\{0,1\}$ is a boolean function defined on index set $I:=[n]$ that is neither monotone nor anti-monotone.

We start off by arguing that $\lim _{k \rightarrow \infty} b s\left(f^{(k)}\right)^{1 / k}$ exists. In order to do this, we need the following simple claim.

Lemma 28. Let $f$ be an n-variate boolean function that is neither monotone nor antimonotone. For all $k \geq 0$ and $b \in\{0,1\}$, we have

$$
\begin{gathered}
b s\left(f^{(k)}\right) \leq b s_{b}\left(f^{(k+1)}\right) \leq n \cdot b s\left(f^{(k)}\right) \\
b s^{*}\left(f^{(k)}\right) \leq b s_{b}^{*}\left(f^{(k+1)}\right) \leq n \cdot b s^{*}\left(f^{(k)}\right)
\end{gathered}
$$

(Here $f^{(0)}$ denotes the univariate identity function.) In particular, for $k \geq 1$, we have $\min \left\{b s_{0}\left(f^{(k)}\right), b s_{1}\left(f^{(k)}\right)\right\} \geq b s\left(f^{(k)}\right) / n$ and similarly for the fractional block sensitivity.

Note that the hypothesis that $f$ is non-monotone is essential. If $f$ is the $n$-variate OR function then $b s_{0}\left(f^{(k)}\right)=n^{k}$ while $b s_{1}\left(f^{(k)}\right)=1$. The hypothesis that $f$ is not antimonotone is not essential and is included for convenience.

Proof. We only prove the claim for block sensitivity. The case of fractional block sensitivity follows by using the exact same reasoning for fractional block packings.

We start with the first inequality. We show it for the case $b=0$, the case $b=1$ is similar. Let $c \in\{0,1\}$ such that $b s\left(f^{(k)}\right)=b s_{c}\left(f^{(k)}\right)=N$. Fix assignments $\alpha^{0}, \alpha^{1}$ to $I^{(k)}$ so that the selector $\vec{\alpha}:=\left(\alpha^{0}, \alpha^{1}\right)$ is $f^{(k)}$-compatible and moreover, $\alpha^{c}$ satisfies $b s_{\alpha^{c}}\left(f^{(k)}\right)=N$.

Since $f$ is neither monotone nor anti-monotone, we can fix an assignment $x$ to $I$ such that $x \in f^{-1}(0)$ and flipping some index $i$ from $c$ to $1-c$ in $x$ results in an assignment $x^{\prime} \in f^{-1}(1)$. Let $X$ be the assignment to $I^{(k+1)}$ defined by $X=x \circ \vec{\alpha}$. We claim that $b s_{X}\left(f^{(k+1)}\right) \geq N$, which will prove the lower bound.

To see this, note that for any block $B$ belonging to $\mathcal{B}_{\alpha^{c}}\left(f^{(k)}\right)$, we can construct a block $\operatorname{lift}(B) \in$ $\mathcal{B}_{X}\left(f^{(k+1)}\right)$ defined using composition as $\operatorname{lift}(B):=e_{i}\left(B^{j}: j \in I\right)$, where $e_{i}$ is the singleton block $\{i\}$ and $B^{j}=B$ for $j=i$ and $\emptyset$ otherwise. Using this method, any block packing $\mathcal{B}$ in $\mathcal{B}_{\alpha^{b}}\left(f^{(k)}\right)$ may be "lifted" to a block packing $\mathcal{B}^{\prime}=\left\{\operatorname{lift}(B) \mid B \in \mathcal{B}_{\alpha^{b}}\left(f^{(k)}\right)\right\}$ of the same size as $\mathcal{B}$. Hence, $b s_{X}\left(f^{(k+1)}\right) \geq b s_{\alpha^{c}}\left(f^{(k)}\right)=N$, which proves the first inequality.

Next we prove the second inequality. Let $X$ be an assignment to $I^{(k+1)}$; we want to show that $b s_{X}\left(f^{(k+1)}\right) \leq n \cdot b s\left(f^{(k)}\right)$. Let $\mathcal{B}$ be any block packing in $\mathcal{B}_{X}\left(f^{(k+1)}\right)$ of maximum size. We may assume that $\mathcal{B}$ contains minimal blocks only, that is, $\mathcal{B} \subseteq \partial \mathcal{B}_{X}\left(f^{(k+1)}\right)$.

Let $\pi$ denote the mapping from $I^{(k+1)}$ to $I^{k}$ obtained by mapping $i_{1}, \ldots, i_{k+1}$ to $i_{2}, \ldots, i_{(k+1)}$. For $i \in I$, let $U_{i}$ be the set of $i_{1}, \ldots, i_{k+1} \in I^{(k+1)}$ with $i_{1}=i$ and let $X_{i}$ be the assignment to $I^{(k)}$ with $X_{i}\left(j_{1}, \ldots, j_{k}\right)=X\left(i, j_{1}, \ldots, j_{k}\right)$. For each block $B \in \mathcal{B}$, let $B_{i}=B \cap U_{i}$. Since each 
$B \in \mathcal{B}$ is a minimal block for $f^{(k+1)}$ at $X$, it follows that if $B_{i} \neq \emptyset$ then $\pi\left(B_{i}\right)$ is a block for $f^{(k)}$ at $X_{i}$ (otherwise $B-B_{i}$ would be a block for $f^{(k+1)}$ at $X$, contradicting the minimality of $B)$. Let $\mathcal{B}_{i}=\left\{\pi\left(B_{i}\right): B \in \mathcal{B}, B_{i} \neq \emptyset\right\}$. Then $\mathcal{B}_{i}$ is a packing of blocks for $f^{(k)}$ at $X_{i}$. Since for each $B \in \mathcal{B}, B_{i}$ is nonempty for at least one index $i$, we have $\sum_{i}\left|\mathcal{B}_{i}\right| \geq|\mathcal{B}|$. It follows that $n \cdot b s\left(f^{(k)}\right) \geq \sum_{i} b s_{X_{i}}\left(f^{(k)}\right) \geq b s_{X}\left(f^{(k+1)}\right)$, as required.

Lemma 29. The limit $\lim _{k \rightarrow \infty} b s\left(f^{(k)}\right)^{1 / k}$ exists and is finite.

Proof. It clearly suffices to show that $\lim _{k \rightarrow \infty} \log \left(b s\left(f^{(k)}\right)\right) / k$ exists and is finite. Finiteness is trivial, since $1 \leq b s\left(f^{(k)}\right) \leq n^{k}$ and hence the sequence $\log \left(b s\left(f^{(k)}\right)\right) / k$ is bounded. To show that the limit exists, we use Corollary 24. To show that $\left\{\log \left(b s\left(f^{(k)}\right)\right)\right\}_{k}$ satisfies the hypothesis of Corollary 24, it suffices to show that $b s\left(f^{(k+\ell)}\right)=\Omega\left(b s\left(f^{(k)}\right) b s\left(f^{(\ell)}\right)\right)$, where the constant in the $\Omega(\cdot)$ is independent of $k$ (but may depend on $n$ ). But by Lemma 26, we have $b s\left(f^{(k+\ell)}\right) \geq b s\left(f^{(k)}\right) \cdot M$, where $M=\min \left\{b s_{0}\left(f^{(\ell)}\right), b s_{1}\left(f^{(\ell)}\right)\right\}$. By Lemma 28, we have $M \geq b s\left(f^{(\ell)}\right) / n$ and thus, it follows that $b s\left(f^{(k+\ell)}\right) \geq b s\left(f^{(k)}\right) b s\left(f^{(\ell)}\right) / n$ and therefore, by Corollary 24, we are done.

Lemma 29 is useful since we can now analyze the limit of an arbitrary subsequence of the sequence $\left\{b s\left(f^{(k)}\right)^{1 / k}\right\}_{k}$ that we are actually interested in.

We now proceed to the proof of Theorem 22. We will need that $\min \left\{b s_{0}\left(f^{(k)}\right), b s_{1}\left(f^{(k)}\right)\right\} \rightarrow \infty$ as $k \rightarrow \infty$. By Corollary 27, this holds whenever $\min \left\{b s_{0}(f), b s_{1}(f)\right\} \geq 2$. We now look at what happens when this is not the case. Without loss of generality assume that $b s_{0}(f)=1$ (since $f$ is nonmonotone and hence non-constant, we have $\left.\min \left\{b s_{0}(f), b s_{1}(f)\right\} \geq 1\right)$. It can be checked that this happens if and only if $f$ is a conjunction of literals. Since $f$ is neither monotone nor anti-monotone, there must be at least one positive and one negative literal. In this case, it can be checked that $\min \left\{b s_{0}\left(f^{(2)}\right), b s_{1}\left(f^{(2)}\right)\right\} \geq 2$. Thus, by Corollary 27, we see that $\min \left\{b s_{0}\left(f^{(2 k)}\right), b s_{1}\left(f^{(2 k)}\right)\right\} \geq 2^{k}$ and by Lemma 28, we have $\min \left\{b s_{0}\left(f^{(2 k+1)}\right), b s_{1}\left(f^{(2 k+1)}\right)\right\} \geq b s\left(f^{(2 k)}\right) \geq 2^{k}$. It follows that $\min \left\{b s_{0}\left(f^{(k)}\right), b s_{1}\left(f^{(k)}\right)\right\} \rightarrow \infty$ as $k \rightarrow \infty$.

Let $L$ denote $\lim _{k \rightarrow \infty} b s^{*}\left(f^{(k)}\right)^{1 / k}$. As $b s\left(f^{(k)}\right) \leq b s^{*}\left(f^{(k)}\right)$ for each $k \geq 1$, we have $\lim _{k \rightarrow \infty} b s\left(f^{(k)}\right)^{1 / k} \leq L$. We now show that for any $\varepsilon \in(0,1)$, it is the case that $\lim _{k \rightarrow \infty} b s\left(f^{(k)}\right)^{1 / k} \geq L(1-\varepsilon)$.

Fix any $\varepsilon \in(0,1)$. Let $\ell_{0} \in \mathbb{N}$ be chosen large enough so that $F=f^{\left(\ell_{0}\right)}$ satisfies the following conditions:

- $b s^{*}(F) \geq(L(1-\varepsilon / 4))^{\ell_{0}}$,

- $n^{-1 / \ell_{0}} \geq(1-\varepsilon / 2)$.

We will show that $\lim _{k \rightarrow \infty} b s\left(F^{(k)}\right)^{1 / k \ell_{0}} \geq L(1-\varepsilon)$. Since $\lim _{k \rightarrow \infty} b s\left(f^{(k)}\right)^{1 / k}=$ $\lim _{k \rightarrow \infty} b s\left(F^{(k)}\right)^{1 / k \ell_{0}}$, this will conclude the proof of Theorem 22 .

Recall from Section 2.6 that for any assignment $z$ to the variables of $F, b s_{z}^{*}(F)=$ $\lim _{M \rightarrow \infty} b s_{z}^{M}(F) / M$. Thus, there exists an $m$ such that for any $M \geq m, b s^{M}(F) \geq$ $M(L(1-\varepsilon / 2))^{\ell_{0}}$. Since $\min \left\{b s_{0}\left(F^{(k)}\right), b s_{1}\left(F^{(k)}\right)\right\} \rightarrow \infty$ as $k \rightarrow \infty$, there exists $k_{0} \in \mathbb{N}$ s.t. $\min \left\{b s_{0}\left(F^{(k)}\right), b s_{1}\left(F^{(k)}\right)\right\} \geq m$ for each $k \geq k_{0}$.

By Lemma 26, for $k \geq k_{0}$, we have $b s\left(F^{(k+1)}\right) \geq b s^{M}(F)$, where $M=\min \left\{b s_{0}\left(F^{(k)}\right), b s_{1}\left(F^{(k)}\right)\right\}$. Since $M \geq m$ by our choice of $k_{0}$ we know that $b s^{M}(F) \geq M(L(1-\varepsilon / 2))^{\ell_{0}}$. Moreover, by Lemma 28, we know that $\min \left\{b s_{0}\left(F^{(k)}\right), b s_{1}\left(F^{(k)}\right)\right\}=\min \left\{b s_{0}\left(f^{\left(\ell_{0} k\right)}\right), b s_{1}\left(f^{\left(\ell_{0} k\right)}\right)\right\} \geq b s\left(F^{(k)}\right) / n$. Thus, we 
have for $k \geq k_{0}, b s\left(F^{(k+1)}\right) \geq(L(1-\varepsilon / 2))^{\ell_{0}} \cdot b s\left(F^{(k)}\right) / n$. Iterating this inequality we obtain for any $k \geq k_{0}$,

$$
\begin{aligned}
b s\left(F^{(k)}\right) & \geq(L(1-\varepsilon / 2))^{\ell_{0}\left(k-k_{0}\right)} \cdot b s\left(F^{\left(k_{0}\right)}\right) / n^{k-k_{0}} \\
& \geq \frac{(L(1-\varepsilon / 2))^{\ell_{0} k}}{C \cdot n^{k}}
\end{aligned}
$$

where $C>0$ is some quantity that is independent of $k$. Thus, we have

$$
\begin{aligned}
\lim _{k \rightarrow \infty} b s\left(F^{(k)}\right)^{1 / k \ell_{0}} & \geq \frac{(L(1-\varepsilon / 2))}{n^{1 / \ell_{0}}} \\
& \geq L(1-\varepsilon / 2)^{2} \geq L(1-\varepsilon)
\end{aligned}
$$

The second inequality above follows since $n^{-1 / \ell_{0}} \geq(1-\varepsilon / 2)$. Thus, we have shown that $\lim _{k \rightarrow \infty} b s\left(f^{(k)}\right)^{1 / k}=\lim _{k \rightarrow \infty} b s\left(F^{(k)}\right)^{1 / k \ell_{0}} \geq L(1-\varepsilon)$. Since $\varepsilon>0$ can be made arbitrarily small, this shows that $\lim _{k \rightarrow \infty} b s\left(f^{(k)}\right)^{1 / k} \geq L$ and concludes the proof of Theorem 22.

\subsection{Correcting a previous separation result}

We use Theorem 22 to correct and clarify a couple of remarks from Aaronson's paper Aar08, Section 5].

Aaronson considers a function $f:\{0,1\}^{6} \rightarrow\{0,1\}$ due to Bublitz et al. [BSW86] for the purposes of creating some separating examples. A short description of the function follows (the function is defined slightly differently by Bublitz et al.). The function $f\left(x_{1}, \ldots, x_{6}\right)$ is defined as the following depth 2 decision tree with parity gates: First compute $x_{1} \oplus x_{2} \oplus x_{3} \oplus x_{4}$, if 0 then output $x_{1} \oplus x_{2} \oplus x_{5}$, else output $x_{1} \oplus x_{3} \oplus x_{6}$. It can be checked that $f$ has the following property (the proof of which is omitted):

Lemma 30. For every $z \in\{0,1\}^{6}, b s_{z}(f)=4, b s_{z}^{*}(f)=C_{z}^{*}(f)=4.5$, and $C_{z}(f)=5$.

1. It is claimed that $b s\left(f^{(k)}\right)=4^{k}$ and $C\left(f^{(k)}\right)=5^{k}$ and thus $C\left(f^{(k)}\right)=b s\left(f^{(k)}\right)^{\log _{4} 5}$ for every $k \in$ $\mathbb{N}$. However, it follows from Theorem 22 that for a boolean function $g, \lim _{k \rightarrow \infty}\left(b s\left(g^{(k)}\right)\right)^{1 / k}=$ $\lim _{k \rightarrow \infty}\left(b s^{*}\left(g^{(k)}\right)\right)^{1 / k}$ as $k \rightarrow \infty$, which may in general be significantly larger than $b s(f)^{k}$. In this case, by Lemma 30 and Theorem 15, it follows that $\left(b s^{*}\right)^{\lim }(f)=4.5$ and hence, by Theorem 22, for any $\varepsilon>0$ and large enough $k \in \mathbb{N}$ depending on $\varepsilon, b s\left(f^{(k)}\right) \geq(4.5-\varepsilon)^{k}$. In particular, this example only yields $\operatorname{crit}(C, b s) \geq \log _{4.5} 5$, which is smaller than the $\log _{4} 5$ separation claimed.

2. It is also claimed that the family $f^{(k)}$ yields polynomial separations between the block sensitivity $b s(\cdot)$ and $R C(\cdot)$, where $R C(F)$ for any boolean function $F$ is the randomized certificate complexity of $f$ (see Section (A)). However, by Theorem 22, it follows that such an approach (irrespective of the base function $f$ ) can never yield a polynomial gap between $b s(\cdot)$ and $R C(\cdot)$, since

$$
b s^{\lim }(f)=\left(C^{*}\right)^{\lim }(f)=\lim _{k \rightarrow \infty}\left(R C\left(f^{(k)}\right)\right)^{1 / k}
$$

where the last equality follows from Claim 38 . 


\section{$5 \quad$ Separating examples}

In this section we prove a tight lower bound of 2 on the critical exponent for $C(f)$ and $b s^{*}(f)$ (and the same tight lower bound holds for the critical exponent for $C(f)$ and $b s^{*}(f)$.) We exhibit two different families of boolean functions that attain this separation. We also exhibit a family of boolean functions that proves a lower bound of $3 / 2$ on the critical exponent of $b s^{*}(f)$ and $b s(f)$.

One of our examples uses iterated composition. The other two examples are obtained by composing the $n$-bit OR function $O R_{n}$ with a suitable function $g$. We will need the following simple fact:

Proposition 31. Let $g$ be a non-constant boolean function and $f=O R_{n} \circ g$. Then for complexity measure $m \in\left\{C, b s, b s^{*}\right\}$ we have:

$$
\begin{aligned}
& m_{1}(f)=m_{1}(g) \\
& m_{0}(f)=n \cdot m_{0}(g) .
\end{aligned}
$$

Proof. Let $I$ be the index set for the variables of $g$, so $J=[n] \times I$ is the index set for the variables of $f$. For $i \in[n]$, write $J_{i}$ for the index subset $\{i\} \times I$.

First we show $m_{1}(f)=m_{1}(g)$. The function $g$ is a subfunction of $f$ (i.e., can be obtained from $f$ by restricting some variables) so $m_{1}(f) \geq m_{1}(g)$ for each of the above complexity measures $m$. For the reverse inequality, we argue that $C_{1}(f) \leq C_{1}(g)$, the argument for the other two measures is similar. Let $\alpha \in g^{-1}(1)$ be an input for which $C_{\alpha}(g)$ is maximum. Construct an input $\beta$ for $f$ by fixing the variables in $J_{n}$ according to $\alpha$ and for each $i \in[n-1]$ fix the variables in $J_{i}$ to some input $y$ for $g$ such that $g(y)=0$. It is easy to check that $C_{1}(f) \leq C_{\beta}(f)=C_{\alpha}(g)=C_{1}(g)$.

Next we show that $m_{0}(f)=n \cdot m_{0}(g)$. For this, write an assignment to the variables of $f$ as $\alpha^{1}, \ldots, \alpha^{n}$ where each $\alpha^{i}$ is an assignment to the variables of $g$. We have $f\left(\alpha^{1}, \ldots, \alpha^{n}\right)=0$ if and only if $g\left(\alpha^{1}\right)=\cdots=g\left(\alpha^{n}\right)=0$. It is easy to check that for each of the measures $m$ under consideration, if $g\left(\alpha^{1}\right)=\cdots=g\left(\alpha^{n}\right)=0$ then $m_{\alpha^{1}, \ldots, \alpha^{n}}(f)=m_{\alpha^{1}}(g)+\cdots+m_{\alpha^{n}}(g)$. Thus an input in $f^{-1}(0)$ that maximizes $m_{\alpha^{1}, \ldots, \alpha^{n}}(f)$ is one for which $\alpha^{1}=\cdots=\alpha^{n}=\alpha$, where $\alpha$ satisfies $m_{0}(g)=m_{\alpha}(g)$. This gives $m_{0}(f)=n \cdot m_{0}(g)$.

\subsection{Achieving quadratic separation between $C(f)$ and $b s(f)$}

\subsubsection{A Probabilistic Construction}

In this section we construct a sequence of $n$-variate functions $g_{n}$ (for $n$ sufficiently large) such that $C_{0}\left(g_{n}\right)=\Omega(n)$ and $b s_{0}\left(g_{n}\right)=O(1)$. We then define $f_{n}=O R_{n} \circ g_{n}$. By Proposition 31, we have $C\left(f_{n}\right) \geq C_{0}\left(f_{n}\right)=n \cdot C_{0}\left(g_{n}\right)=\Omega\left(n^{2}\right)$, while $b s\left(f_{n}\right) \leq b s^{*}\left(f_{n}\right) \leq \max \left(b s_{0}^{*}\left(f_{n}\right), b s_{1}^{*}\left(f_{n}\right)\right) \leq$ $\max \left(n b s_{0}^{*}\left(g_{n}\right), b s_{1}^{*}\left(g_{n}\right)\right)=O(n)$.

This will prove:

Theorem 32. For every $n \in \mathbb{N}$ sufficiently large, there is a function $f:\{0,1\}^{n^{2}} \rightarrow\{0,1\}$ such that $b s(f) \leq b s^{*}(f)=O(n)$ and $C(f)=\Omega\left(n^{2}\right)$.

Let us write $\delta(x, y)$ to denote the Hamming distance between $x, y \in\{0,1\}^{n}$. We define $g=$ $g_{n}:\{0,1\}^{n} \rightarrow\{0,1\}$ as follows (we view $n$ as being sufficiently large). Choose $x_{1}, \ldots, x_{N} \in\{0,1\}^{n}$ 
uniformly at random (with replacement) with $N=2^{n / 50}$. We set $g\left(x_{i}\right)=1$ for each $i$, and $g(x)=0$ otherwise.

Claim 33. With high probability, for all $i, j$ distinct $\delta\left(x_{i}, x_{j}\right) \geq \frac{n}{100}$.

Proof. Let $A_{i, j}$ denote the event $\delta\left(x_{i}, x_{j}\right)<\frac{n}{100}$. Let $x$ be a fixed point in $\{0,1\}^{n}$ and $B(x, r)$ denote the Hamming ball of radius $r$ and center $x$. Then $|B(x, r)|=\sum_{i=0}^{r}\left(\begin{array}{c}n \\ i\end{array}\right)$. Thus we have

$$
B\left(x, \frac{n}{100}\right)<2\left(\begin{array}{c}
n \\
n / 100
\end{array}\right) \leq 2(100 e)^{n / 100}<2^{n / 10}
$$

These inequalities imply that

$$
\mathbf{P}\left(A_{i, j}\right)=\frac{B\left(x, \frac{n}{100}\right)}{2^{n}}<2^{-9 n / 10} .
$$

By the union bound the hypothesis fails with probability at most

$$
2^{-9 n / 10}\left(\begin{array}{c}
N \\
2
\end{array}\right)=o(1)
$$

If the hypothesis of the claim holds and $g(x)=0$, then all but possibly one of the blocks for $g$ at $x$ will have size at least $\frac{n}{200}$. Thus, at most 200 blocks can be packed and $b s_{0}(g) \leq 200$. Likewise, this bound on the size of blocks implies that $b s_{0}^{*}(g) \leq 200$.

We now argue that all sufficiently large subcubes of $\{0,1\}^{n}$ will contain a 1 of $g$ almost surely.

Claim 34. With high probability, $C_{0}(g) \geq \frac{n}{100}$

Proof. Its enough to show that every subcube of co-dimension $\frac{n}{100}$ will contain a $y$ such that $g(y)=1$. For each $S$ which is a subcube of co-dimension $\frac{n}{100}$, denote $A_{S}$ as the event $g(x)=0$ for all $x \in S$. Then

$$
\mathbf{P}\left(A_{S}\right) \leq\left(1-2^{-n / 100}\right)^{N}<\exp \left(-\frac{N}{2^{n / 100}}\right)=\exp \left(-2^{n / 100}\right)
$$

There are $\left(\begin{array}{c}n \\ n / 100\end{array}\right) 2^{n / 100}<2^{2 n}$ subcubes of co-dimension $\frac{n}{100}$. Thus by union bound the hypothesis fails with probability at most

$$
\exp \left(-2^{n / 100}\right) 2^{2 n}=o(1)
$$

We have shown, for sufficiently large $n$, that with high probability a random function $g$ satisfies $b s_{0}^{*}(g) \leq 200$ and $C_{0}(g) \geq \frac{n}{100}$. Thus for each $n$ sufficiently large, there exists a function $g_{n}$ with this property. 


\subsubsection{A Construction Using Iterated Composition}

In this section we construct a function $f$ on $n$ variables for which $C^{\lim }(f) \geq \frac{n}{2}$ and $\left(C^{*}\right)^{\lim }(f) \leq$ $4 \sqrt{n}$. For any $\epsilon>0$, we may choose $n$ large enough to conclude that $\operatorname{crit}\left(C^{*}, C\right) \geq 2-\epsilon$.

Let $d, k, n$ be positive integers such that $n \geq k \geq d, d \mid k$, and $k \mid n$. We define $f:\{0,1\}^{n} \rightarrow$ $\{0,1\}$ to be the following boolean function on $n$ variables:

View the $n$ indices of the input $x$ as being divided into $\frac{n}{k}$ disjoint groups, with each group containing $k$ indices. $f$ accepts if and only if $|x| \geq d$ and all the 1's in $x$ can be found in a single group. Note that $f(x)=1$ implies $|x| \leq k$.

Although $f$ shows no separation between $b s(f)$ and $C(f)$, the key is that both the zero and one certificate complexity for $f$ are large, while the zero block sensitivity is small. Also, any 1 -assignment for $f$ contains many 0 indices.

In the following analysis, we assume $n$ is an even perfect square and set $k:=2 \sqrt{n}$ and $d:=\sqrt{n}$. We wish to bound $C^{l i m}(f)$ and $\left(C^{*}\right)^{\lim }(f)$. By Theorem [15, it is enough to bound $\widehat{C}(f)$ and $\widehat{C^{*}}(f)$ instead.

Claim 35. For the boolean function $f:\{0,1\}^{n} \rightarrow\{0,1\}$ defined above we have:

$$
\widehat{C^{*}}(f) \leq 4 \sqrt{n} \text {. }
$$

Proof. We proceed by showing that for any assignment selector $\vec{\alpha}=\left(\alpha^{0}, \alpha^{1}\right)$, we can find a pair of hitting sets $\left(w^{0}, w^{1}\right)$ such that the corresponding profile matrix has all eigenvalues less than $4 \sqrt{n}$. We look at the 0 assignments first, and for each possible $\alpha^{0}$ we exhibit a small fractional hitting set $w_{0}$.

Case 1, $\alpha^{0}=(0,0, \ldots, 0)$ :

Here we choose $w_{0}:=\left(\frac{1}{d}, \frac{1}{d}, \cdots, \frac{1}{d}\right)$. It follows that, $w_{0}$ is a fractional hitting set as each block for this assignment has size at least $d$. For this hitting set the profile vector $p_{\alpha^{0}}\left(w_{0}\right)=\left(\frac{n}{d}, 0\right)$.

Case 2, $\left|\alpha^{0}\right|=j$, and all 1's in $\alpha^{0}$ appear in the same group:

Note this means that $j<d$ as $\alpha^{0}$ is a 0 assignment. Let $X_{1}$ be the set of indices for $\alpha^{0}$ which are 1's, let $G_{1}$ be the group which contains $X_{1}$. Pick an $s \in X_{1}$, we define a fractional hitting set $w_{0}$ to assign weight 1 to $s$, weight 1 to all indices in $G_{1} \backslash X_{1}$, and weight 0 otherwise. To see that $w_{0}$ is indeed a hitting set, note that if $B$ is a block for $\alpha^{0}$, then either $B \subseteq G_{1}$ or $X_{1} \subset B$. If $X_{1} \subset B$, then $s \in B$ and it has been assigned weight 1 . If $B \subseteq G_{1}$ then $B$ must contain a 0 index in $G_{1}$ as $\left|\alpha^{0}\right|<d$, this index was assigned weight 1 by $w_{0}$. Thus $w_{0}$ is a hitting set and the profile vector $p_{\alpha^{0}}\left(w_{0}\right)=(k-j, 1) \leq(k, 1)$.

Case 3, At least two different groups in $\alpha^{0}$ contain 1's:

Let $G_{1}, G_{2}$ be two distinct groups containing 1's. Let $X_{1}, X_{2}$ be the set of indices which are assigned 1 by $\alpha^{0}$ in $G_{1}, G_{2}$ respectively. Then if $B$ is a block for $\alpha^{0}$, either $X_{1} \subseteq B$ or $X_{2} \subseteq B$. We define $w_{0}$ to assign weight 1 to an index in $X_{1}$ and in index in $X_{2}$. This will be a hitting set, and the profile vector $p_{\alpha^{0}}\left(w_{0}\right)=(0,2)$. This concludes the analysis of each possible 0 assignment.

The 1 assignments $\alpha^{1}$ :

If $\alpha^{1}$ is a 1 assignment then $\left|\alpha^{1}\right| \geq d$ and all the 1 's appear in a single group, call it $G_{1}$. In this case we define $w_{1}$ to assign weight 1 to all indices outside $G_{1}$, and weight 1 to $d$ indices in $G_{1}$ which are assigned 1 by $\alpha^{1}$. This will be a hitting set as any block must contain a 0 index outside of $G_{1}$ or leave less than $d$ 1's inside of $G_{1}$ after flipping the indices in $B$. Here the profile vector $p_{\alpha^{1}}\left(w_{1}\right)=(n-k, d)$. 
If $M, M^{\prime}$ are $2 \times 2$ matrices with nonnegative entries, and $M \leq M^{\prime}$ entry by entry, then $\rho(M) \leq \rho\left(M^{\prime}\right)$. Considering this along with the 3 cases of 0 assignments above, bounding $\widehat{C^{*}}(f)$ reduces to bounding the largest eigenvalues of the following matrices:

$$
\left[\begin{array}{ll}
\frac{n}{d} & 0 \\
n-k & d
\end{array}\right] \quad\left[\begin{array}{cc}
k & 1 \\
n-k & d
\end{array}\right] \quad\left[\begin{array}{cc}
0 & 2 \\
n-k & d
\end{array}\right]
$$

Here the second matrix has the largest eigenvalue of the three. It is easy to check that $k=2 \sqrt{n}$, $d=\sqrt{n}$ implies its largest eigenvalue is less than $4 \sqrt{n}$.

\section{Claim 36.}

$$
\widehat{C}(f) \geq \frac{n}{2}
$$

Proof. To prove this we choose an assignment selector $\vec{\alpha}$ for which all profile matrices $A \in \mathcal{M}_{\vec{\alpha}}(f)$ have an eigenvalue larger than $\frac{n}{2}$. We set $\alpha^{0}:=(0,0, \cdots, 0)$ and $\alpha^{1}$ to have exactly $d$ 's in the first group, and be identically 0 in every other group.

Any certificate for $\alpha^{0}$ must fix $k-d+1$ indices in each group, thus must fix $\frac{n}{k}(k-d+1)$ in total. It follows that any minimum certificate $w_{0}$ (viewed as a boolean valued weight function) yields the profile vector $p_{\alpha^{0}}\left(w_{0}\right)=\left(\frac{n}{k}(k-d+1), 0\right)$.

Likewise, any certificate for $\alpha^{1}$ must fix all 1 indices (there are $d$ of them), and fix all the 0 indices outside the unique group containing the 1's. Thus any minimal profile vector $p_{\alpha^{1}}\left(w_{1}\right)=(d, n-k)$. The claim then reduces to looking at the maximum eigenvalue of the matrix

$$
A=\left[\begin{array}{cc}
\frac{n}{k}(k-d+1) & 0 \\
n-k & d
\end{array}\right] .
$$

When $k=2 \sqrt{n}$ and $d=\sqrt{n}$ this matrix has an eigenvalue larger than $\frac{n}{2}$.

\subsection{A separation between fractional block sensitivity and block sensitivity}

Theorem 37. For infinitely many natural numbers $n$, there is an $n^{2}$-variate function $f_{n}$ : $\{0,1\}^{n^{2}} \rightarrow\{0,1\}$ s.t. $b s\left(f_{n}\right)=O(n)$ and $b s^{*}\left(f_{n}\right)=\Omega\left(n^{3 / 2}\right)$. Therefore $\operatorname{crit}\left(b s^{*}, b s\right) \geq 3 / 2$.

To construct $f_{n}$, we build an $n$-variate function $g=g_{n}:\{0,1\}^{n} \rightarrow\{0,1\}$ satisfying $b s_{0}(g)=$ $O(1)$ and $b s_{0}^{*}(g)=\Omega(\sqrt{n})$. We then define $f_{n}=O R_{n} \circ g$. Using Proposition 31] we conclude that $b s\left(f_{n}\right)=O(n)$ and $b s^{*}(g)=\Omega\left(n^{3 / 2}\right)$. (In a previous version of this paper our construction for the function $g$ was random, and gave a weaker bound of $\Omega(\sqrt{n / \log n})$ for $b s_{0}^{*}(g)$, which was still enough to show $\operatorname{crit}\left(b s^{*}, b s\right) \geq 3 / 2$. Avishay Tal (personal communication) gave an alternate explicit construction which gave the bound of the theorem, which is what we present here.)

The function $g=g_{n}$ is defined for any $n$ of the form $\left(\begin{array}{l}s \\ 2\end{array}\right)$ for an integer $s$. Identify the input bits of $g$ with the edges of the complete graph $K_{s}$. An assignment $\alpha$ to the variables of $g$ can be viewed as an undirected graph $G_{\alpha}$ consisting of those edges assigned 1 by $\alpha$. We denote by $H_{i}$ the star centered at vertex $i$ and by $x^{i}$ the corresponding input in $\{0,1\}^{I_{1}}$. The function $g(x)$ is defined to be 1 iff $x=x^{i}$ for some $i \in[n]$.

We now show that $g$ satisfies:

(a) $g\left(0^{n}\right)=0$, 
(b) $b s_{0^{n}}^{*}(g) \geq s / 2=\Theta(\sqrt{n})$,

(c) $b s_{0}(g) \leq 3$.

Property (a) is immediate. For property (b), note that the blocks for $g$ at $0^{s}$ are the stars $H_{i}$, and each edge appears in exactly 2 of these stars, so putting weight $1 / 2$ on each of these stars gives a fractional packing of blocks of total weight $s / 2$.

We now prove property (c). Fix any assignment $a \in g^{-1}(0)$ and let $G_{a}$ denote the corresponding graph. We show that $b s_{a}(g) \leq 3$. Assume, for the sake of contradiction, that $b s_{a}(g) \geq 4$. Then, there exists four edge-disjoint graphs $J_{1}, J_{2}, J_{3}$, and $J_{4}$ such that starting from $a$ and flipping all the bits indexed by $J_{\ell}$ (for any $\ell \in[4]$ ) produces one of the graphs $H_{i}$. By renaming input bits if necessary, we may assume that the star graphs thus produced are $H_{1}, H_{2}, H_{3}$, and $H_{4}$ respectively. Thus $J_{i}=G_{a} \oplus H_{i}$, where $\oplus$ denotes symmetric difference. Since edge $\{1,2\}$ belongs to $H_{1}$ and $H_{2}$ we must have $\{1,2\} \in G_{a}$ so that $J_{1}$ and $J_{2}$ are disjoint. But then $\{1,2\} \in J_{3} \cap J_{4}$, contradicting their disjointness.

\section{Acknowledgements}

We would like to thank Avishay Tal for his permission to include his separating example used in the proof of Theorem 37 .

\section{References}

[Aar08] Scott Aaronson. Quantum certificate complexity. J. Comput. Syst. Sci., 74(3):313-322, 2008 .

[BdW02] Harry Buhrman and Ronald de Wolf. Complexity measures and decision tree complexity: a survey. Theor. Comput. Sci., 288(1):21-43, 2002.

[BSW86] Siegfried Bublitz, Ute Schurfeld, and Ingo Wegener. Properties of complexity measures for PRAMs and WRAMs. Theor. Comput. Sci., 48(1):53-73, 1986.

[HKP11] Pooya Hatami, Raghav Kulkarni, and Denis Pankratov. Variations on the Sensitivity Conjecture. Number 4 in Graduate Surveys. Theory of Computing Library, 2011.

[Mey00] Carl D. Meyer. Matrix analysis and applied linear algebra. Society for Industrial and Applied Mathematics, Philadelphia, PA, USA, 2000.

[Mid04] G. Midrijanis. Exact quantum query complexity for total boolean functions. arXiv preprint quant-ph/0403168, 2004.

[Nis91] Noam Nisan. CREW PRAMs and Decision Trees. SIAM J. Comput., 20(6):999-1007, 1991.

[SUB11] E.R. Scheinerman, D.H. Ullman, and C. Berge. Fractional Graph Theory: A Rational Approach to the Theory of Graphs. Dover Books on Mathematics Series. Dover Publications, 2011. 
[Tal12] Avishay Tal. Properties and applications of boolean function composition. Electronic Colloquium on Computational Complexity (ECCC), 19:163, 2012.

[Tal13] Avishay Tal. Properties and applications of boolean function composition. In ITCS, pages 441-454, 2013.

\section{A Fractional Certificate complexity vs. Randomized Certificate complexity}

In Aar08, Aaronson introduced the notion of the Randomized Certificate complexity of a boolean function $g:\{0,1\}^{n} \rightarrow\{0,1\}$.

For $g$ as above and an input $z \in\{0,1\}^{n}$, a Randomized Verifier for $z$ is a non-adaptive randomized query algorithm that expects an $n$-bit input $z^{\prime}$ and behaves as follows 1 The query algorithm queries each bit $z_{i}^{\prime}$ of its input independently with some fixed probability $\lambda_{i} \in[0,1]$ and accepts iff it finds no disagreement between $z^{\prime}$ and $z$. Moreover, the query algorithm satisfies the following soundness property: given any $z^{\prime}$ s.t. $g\left(z^{\prime}\right) \neq g(z)$, it rejects $z^{\prime}$ with probability at least $1 / 2$.

The cost of such a verifier is the expected number of bits of $z^{\prime}$ that are queried, which is $\sum_{i \in[n]} \lambda_{i}$. The Randomized Certificate complexity of $f$ at $z$ is defined to be $R C^{z}(f):=$ $\min \{c \mid$ There is a cost $c$ verifier for $z\}$. The Randomized Certificate complexity of $f$ is defined to be $R C(f):=\max _{z \in\{0,1\}^{n}} R C^{z}(f)$.

The following relation between $R C(g)$ and $C^{*}(g)$ can be proved.

Claim 38. Fix any boolean function $g:\{0,1\}^{n} \rightarrow\{0,1\}$ and any $z \in\{0,1\}^{n}$. Then, $R C^{z}(g)=$ $\Theta\left(C_{z}^{*}(g)\right)$. That is, the quantities $R C^{z}(g)$ and $C_{z}^{*}(g)$ are within a fixed universal constant factor for any $g, z$ as above. In particular, $R C(g)=\Theta\left(C^{*}(g)\right)$.

Proof. We first show that $C_{z}^{*}(g)=O\left(R C^{z}(g)\right)$. Fix an optimal verifier for $z$ and let $\lambda_{i} \in[0,1]$ be the probability that it queries the $i$ th bit of its input $z^{\prime}$. Fix any block $B \in \mathcal{B}_{z}(g)$ and consider the input $z^{\prime}$ obtained by starting with $z$ and flipping the bits indexed by $B$. Since $g\left(z^{\prime}\right) \neq g(z)$, the soundness property of the verifier implies that the verifier probes a bit in $B$ is at least $1 / 2$; in particular, by the union bound, $\sum_{i \in B} \lambda_{i} \geq 1 / 2$.

Consider the function $\sigma:[n] \rightarrow[0,1]$ defined by $\sigma(i):=\min \left\{2 \lambda_{i}, 1\right\}$. The above immediately implies that for any block $B \in \mathcal{B}_{z}(g)$, we have $\sum_{i \in B} \sigma(i) \geq 1$ and hence $\sigma \in \mathcal{W}_{z}^{*}(g)$. Moreover, $|\sigma| \leq 2 \sum_{i} \lambda_{i}=O\left(R C^{z}(g)\right)$ since we considered an optimal verifier for $z$. Thus, $C_{z}^{*}(g) \leq|\sigma|=$ $O\left(R C^{z}(g)\right)$.

We now show that $R C^{z}(g)=O\left(C_{z}^{*}(g)\right)$. Fix an optimal fractional certificate $\sigma \in \mathcal{W}_{z}^{*}(g)$. Consider the randomized query algorithm that queries each bit of its $n$-bit input $z^{\prime}$ with probability $\sigma(i)$ and rejects on finding any disagreement with $z$. To show that this gives us a verifier for $z$, we need to verify the soundness property. Given any input $z^{\prime}$ s.t. $g\left(z^{\prime}\right) \neq g(z)$, the set of indices $B \subseteq[n]$ where $z$ and $z^{\prime}$ differ is a block of $g$ at $z$ and hence, we must have $\sum_{i \in B} \sigma(i) \geq 1$.

Thus, the probability that the verifier accepts $z^{\prime}$ is equal to $\prod_{i \in B}(1-\sigma(i)) \leq$ $\exp \left\{-\sum_{i \in B} \sigma(i)\right\} \leq e^{-1}<1 / 2$. This proves the soundness property of the verifier. Note

\footnotetext{
${ }^{1}$ Strictly speaking, this corresponds to the definition of non-adaptive Randomized Certificate complexity from Aaronson's paper. However, by Lemma 2.1 of [Aar08, it follows that this is within a fixed universal constant of the Randomized Certificate complexity of $f$.
} 
that the expected number of queries made by the verifier is exactly $|\sigma|=C_{z}^{*}(g)$ and hence, $R C^{z}(g) \leq C_{z}^{*}(g)$. 